Aus der Klinik für Kardiologie und Pneumologie

(Prof. Dr. med. G. Hasenfuß)

im Zentrum Innere Medizin

der Medizinischen Fakultät der Universität Göttingen

\title{
Prognostische Wertigkeit des T-Wellen-Alternans bei Patienten mit Erstimplantation eines implantierbaren Kardioverter- Defibrillators
}

\author{
INAUGURAL-DISSERTATION \\ zur Erlangung des Doktorgrades \\ der Medizinischen Fakultät der \\ Georg-August-Universität zu Göttingen
}

\author{
vorgelegt von \\ Frank Hofschulte
}

aus

Hannover

Göttingen 2014 
Dekan:

Prof. Dr. rer. nat. H. K. Kroemer

I. Berichterstatter:

II. Berichterstatter:

III. Berichterstatter:

IV. Berichterstatter:

Tag der mündlichen Prüfung:
Prof. Dr. med. M. Zabel

Prof. Dr. med. T. Paul

PD Dr. med. M. Roessler

Prof. Dr. med. M. Oppermann 
"Reduction of the incidence of SCD depends on identification of those at most risk."

Dr. Rachel C. Myles 


\section{Inhaltsverzeichnis}

Abkürzungsverzeichnis ............................................................................................III

Abbildungs- und Tabellenverzeichnis ..............................................................V

1 Einleitung ........................................................................................... 1

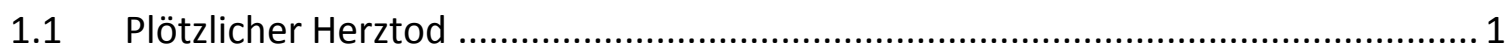

1.2 Implantierbare Kardioverter-Defibrillatoren .................................................... 1

1.3 Studienlage zur präventiven Implantation von ICD-Systemen............................. 2

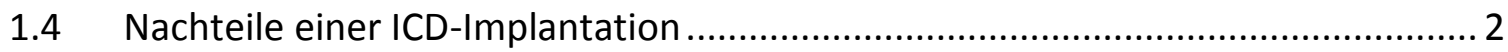

1.5 Apparative Methoden zur Risikostratifikation ................................................ 4

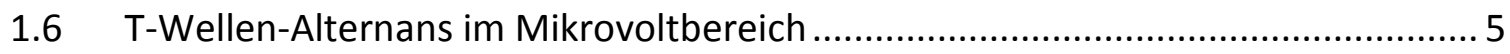

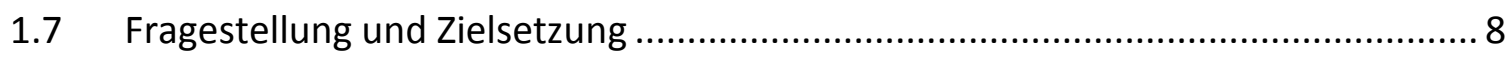

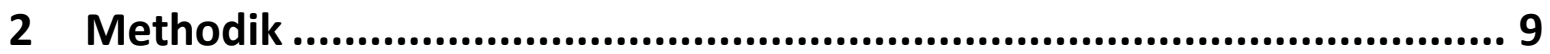

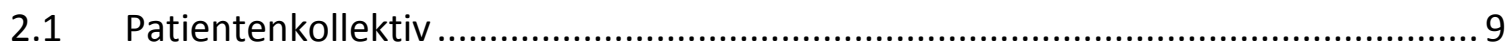

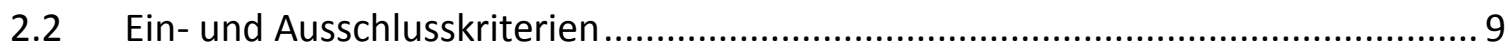

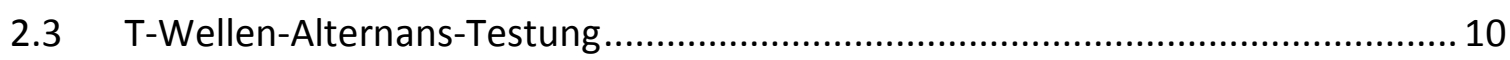

2.3.1 Material und Vorbereitungen für eine TWA-Testung .................................. 10

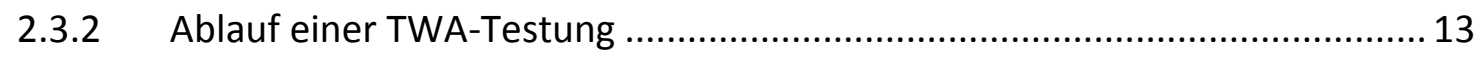

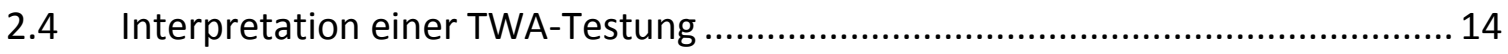

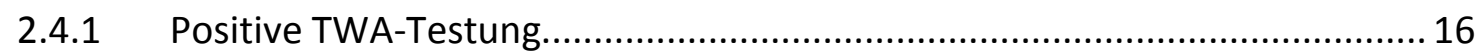

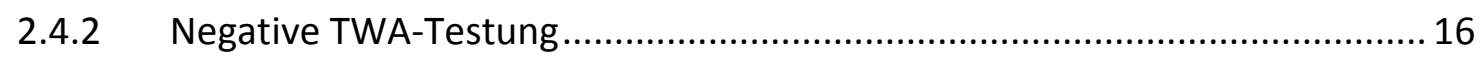

2.4.3 Unbestimmbare TWA-Testung ............................................................... 16

2.4.4 Negative TWA-Testung nach Interpretation mittels B-Kriterien................... 16

2.4.5 Unbestimmbare TWA-Testung nach Interpretation mittels B-Kriterien........ 17

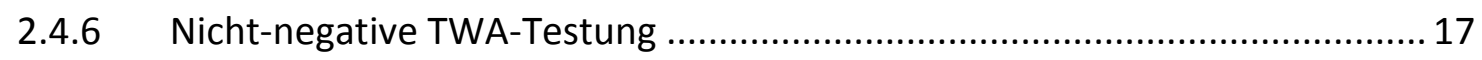

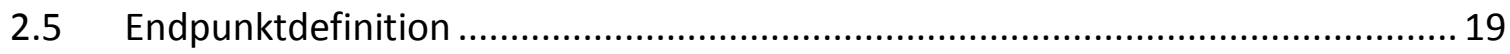

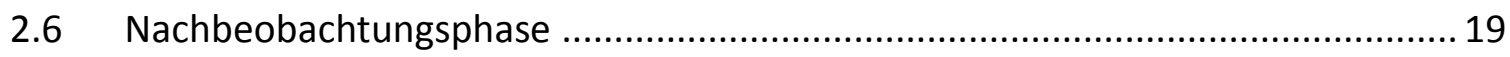

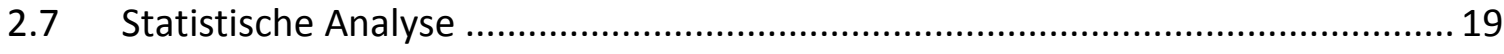

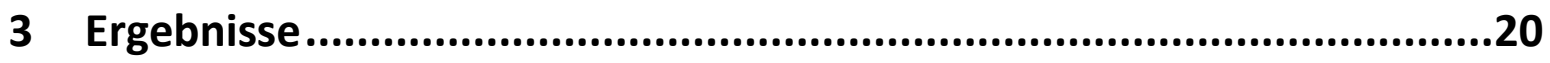

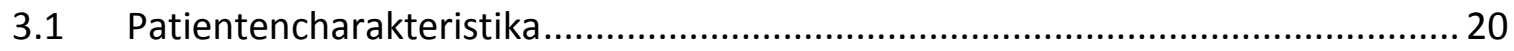

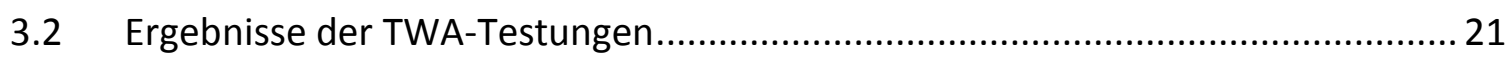




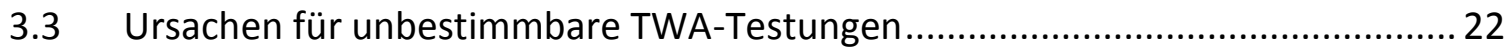

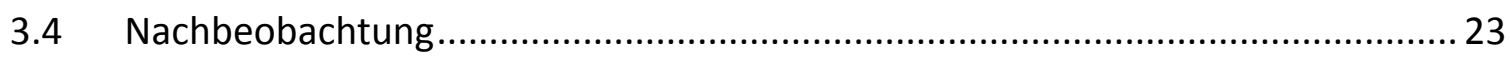

3.5 Ereignisse während der Nachbeobachtungszeit ............................................... 23

3.6 Erreichen eines Endpunktes in Korrelation mit dem Ergebnis der TWA-Testung. 24

3.6.1 Patienten mit appropriater Schockabgabe ................................................. 25

3.6.2 Patienten mit dem primären Endpunkt „Mortalität“ ".................................. 26

3.6.3 Kombinierter Endpunkt „appropriate Schockabgabe“ und „Mortalität“ ...... 29

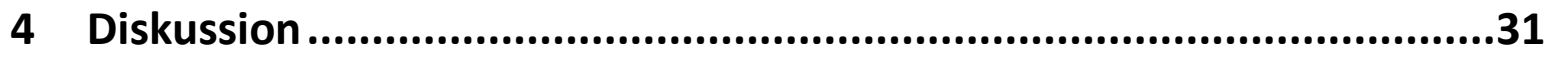

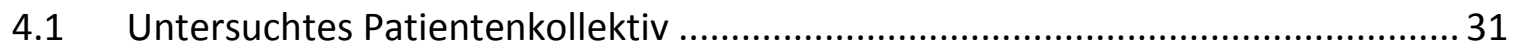

4.2 Verteilung der Testergebnisse im nichtselektierten Patientenkollektiv ............... 32

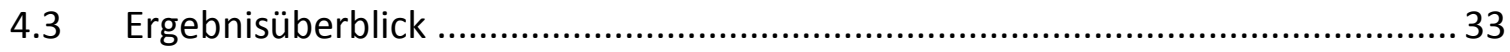

4.4 Bedeutung von unbestimmbaren TWA-Testungen ............................................ 36

4.5 Anwendbarkeit von TWA-Testungen als Risikomarker für den SCD .................... 39

5 Zusammenfassung ...........................................................................42

6 Anhang...............................................................................43

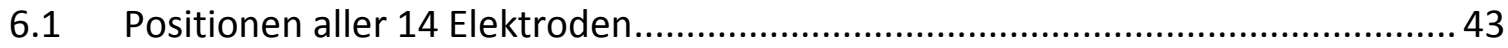

7 Literaturverzeichnis..................................................................44 


\section{Abkürzungsverzeichnis}

$\begin{array}{ll}\text { Abb. } & \text { Abbildung } \\ \text { ACC } & \text { American College of Cardiology } \\ \text { AHA } & \text { American Heart Association } \\ \text { appr. } & \text { appropriat } \\ \text { ATP } & \text { antitachykardes Pacing } \\ \text { bpm } & \text { Schläge pro Minute (beats per minute) } \\ \text { DCM } & \text { dilatative Kardiomyopathie (dilated cardiomyopathy) } \\ \text { EKG } & \text { Elektrokardiographie } \\ \text { EPU } & \text { elektrophysiologische Untersuchung } \\ \text { Erhol } & \text { Erholung } \\ \text { ESC } & \text { Kiloohm } \\ \text { KHK } & \text { ischämische Kardiomyopathie (ischemic cardiomyopathy) } \\ \text { ICD } & \text { Herzfrequenz } \\ \text { HOCM } & \text { hypertrophische obstruktive Kardiomyopathie } \\ \text { HR } & \text { implantierbarer Kardioverter-Defibrillator (implantable cardioverter } \\ \text { ICM } & \end{array}$




\begin{tabular}{|c|c|}
\hline LVEF & linksventrikuläre Ejektionsfraktion \\
\hline $\operatorname{maxNegHR}$ & maximale negative Herzfrequenz \\
\hline $\min$ & Minute (minute) \\
\hline MTWA & T-Wellen-Alternans im Mikrovoltbereich \\
\hline NNT & Anzahl der notwendigen Behandlungen (number needed to treat) \\
\hline npW & negativ prädiktiver Wert \\
\hline ns & nicht signifikant \\
\hline NYHA & New York Heart Association \\
\hline QRS & Komplex im EKG, bestehend aus $\mathrm{Q},-\mathrm{R},-$ und S-Zacke \\
\hline QT & Q-Zacke und T-Welle im EKG \\
\hline SCD & plötzlicher Herztod (sudden cardiac death) \\
\hline Störsig & Störsignale \\
\hline Tab. & Tabelle \\
\hline TWA & T-Wellen-Alternans \\
\hline$U$ & Umdrehungen \\
\hline UMG & Universitätsmedizin Göttingen \\
\hline VF & Kammerflimmern (ventricular fibrillation) \\
\hline vgl. & vergleiche \\
\hline VM & vektorielle Magnitudenableitung (vector magnitude) \\
\hline vs. & versus \\
\hline VT & ventrikuläre Tachykardie \\
\hline$x$ & bipolare EKG-Ableitung im Rahmen der Vektorkardiografie \\
\hline Y & bipolare EKG-Ableitung im Rahmen der Vektorkardiografie \\
\hline Z & bipolare EKG-Ableitung im Rahmen der Vektorkardiografie \\
\hline z. B. & zum Beispiel \\
\hline
\end{tabular}




\section{Abbildungs- und Tabellenverzeichnis}

Abb. 1 Schematische Darstellung eines T-Wellen-Alternans 5

Abb. 2 Elektrodenkonfiguration aller 14 Elektroden (Screenshot) ...................................... 11

Abb. 3 Multikontaktelektrode (Micro-V Alternans Sensor ${ }^{\mathrm{TM}}$ ) ............................................ 12

Abb. 4 Patientenmodul PM-3 ${ }^{\mathrm{TM}}$ der Firma Cambridge Heart, Inc. ..................................... 13

Abb. 5 Beispiel eines TWA-Berichtes von einem 69-jährigen Patienten ............................. 18

Abb. 6 Prozentuale Verteilung der Ergebnisse aller 134 TWA-Testungen .......................... 21

Abb. 7 Verteilung der nicht-negativen gegenüber den negativen Testergebnissen ........... 22

Abb. 8 Anzahl der Ereignisse während des Beobachtungszeitraumes ............................... 23

Abb. 9 Ereignisfreies Überleben (hier: „appropriate Schockabgabe“) in Abhängigkeit von einem negativen, positiven bzw. eines unbestimmbaren Testergebnis

Abb. 10 Ereignisfreies Überleben (hier: „appropriate Schockabgabe“) in Abhängigkeit von einem negativen bzw. eines nicht-negativen Testergebnis

Abb. 11 Überlebenswahrscheinlichkeit in Abhängigkeit vom TWA-Testergebnis („unbestimmbar“, „positiv“ bzw. „negativ“)

Abb. 12 Überlebenswahrscheinlichkeit in Abhängigkeit von einem negativen bzw. eines nicht-negativen Testergebnis

Abb. 13 Ereignisfreies Überleben für den kombinierten Endpunkt „appropriate Schockabgabe" und "Mortalität" in Abhängigkeit vom TWA-Testergebnis („unbestimmbar", „negativ“ bzw. „positiv“)

Abb. 14 Überlebenswahrscheinlichkeit und Wahrscheinlichkeit für eine appropriate Schockabgabe in Abhängigkeit vom Vorhandensein eines negativen bzw. eines nichtnegativen Testergebnisses 30

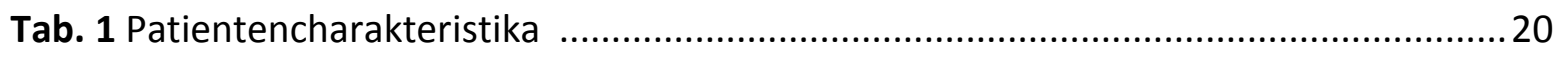

Tab. 2 Patienten mit Erstimplantation ohne TWA-Testung ..............................................2 21

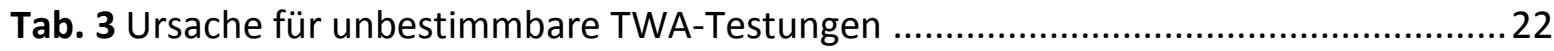

Tab. 4 Erreichen eines Endpunktes in Korrelation mit dem Ergebnis der TWA-Testung .....24

Tab. 5 Verteilung der Testergebnisse der während der Nachbeobachtungszeit verstorbenen Patienten .26

Tab. 6 Elektrodenpositionen aller 14 Elektroden .43 


\section{Einleitung}

\subsection{Plötzlicher Herztod}

Der plötzliche Herztod (sudden cardiac death [SCD]) ist mit einer jährlichen Inzidenz von etwa 80.000 Fällen pro Jahr die häufigste Todesursache in der Bundesrepublik Deutschland (Andresen 2007). Dieser ist definiert als ein natürlicher Tod kardialer Ursache, der binnen einer Stunde nach Beginn akuter Symptome eintritt (Miyazaki et al. 2010; Myerburg und Castellanos 2006). Bayés de Luna et al. konnten 1989 anhand der Auswertung von Langzeit-EKG-Aufzeichnungen während plötzlicher Herztodesfälle zeigen, dass bei mehr als $80 \%$ der Patienten ${ }^{1}$ eine anhaltende ventrikuläre Tachykardie (VT) auftrat, die sekundär in ein Kammerflimmern (VF) überging. Die übrigen Patienten starben an einer bradykarden Rhythmusstörung.

Strukturelle Herzerkrankungen zählen zu den wichtigsten Risikofaktoren und bedingen ein zehnfach erhöhtes Risiko für das Auftreten eines SCD. Annähernd $50 \%$ der von einem SCD betroffenen Patienten haben eine eingeschränkte linksventrikuläre Funktion (Huikuri et al. 2001). Bei der Mehrheit der Patienten stellt zudem eine koronare Herzkrankheit (KHK) die zugrunde liegende Erkrankung dar, gefolgt von dilatativen und hypertrophischen Kardiomyopathien (Greene 1990). Zwar ist die Häufigkeit eines SCD in bestimmten Hochrisikokollektiven relativ hoch, jedoch tritt der SCD in absoluten Zahlen in der Normalbevölkerung deutlich häufiger auf (Myerburg et al. 1998).

\subsection{Implantierbare Kardioverter-Defibrillatoren}

Zur Prävention des SCD stehen neben einer Behandlung der kardialen Grunderkrankung seit Anfang der 1980er Jahre implantierbare Kardioverter-Defibrillatoren (ICDs) zur Verfügung, die durch ein schnelles Eingreifen in den Herzrhythmus hocheffektiv einen SCD verhindern können (Lüderitz 2002; Mirowski 1980). Bei einem ICD handelt es sich um eine Weiterentwicklung eines Herzschrittmachersystems, welches in der Lage ist, bedrohliche Arrhythmien über ein transvenöses Elektrodensystem zu detektieren und zu terminieren (Maisel 2007). Das Aggregat eines ICD wird vergleichbar mit einem Schrittmacheraggregat meist sub- oder epipektoral implantiert (Baddour et al. 2010). Neben den antibradykarden Therapiemöglichkeiten eines konventionellen Herzschrittmachers verfügt ein ICD zudem über die Möglichkeit, ventrikuläre Tachyarrhythmien zu therapieren. Dabei wird die erkannte Rhythmusstörung vom ICD nach einem manuell anpassbaren Algorithmus je nach Höhe der festgestellten Kammerfrequenz als ventrikuläre Tachykardie (VT) oder als Kammerflimmern (VF) klassifiziert. Die zwei wesentlichen Funktionen eines ICD sind einerseits das antitachykarde Pacing (ATP), andererseits die Defibrillation (Wathen et al. 2004). Im Falle einer ventrikulären Tachykardie wird zur Terminierung der Rhythmusstörung ein programmierter Ablauf von gezielten Überstimulationen -als ATP bezeichnet- ausgelöst (Grimm et al. 2006). Statt der Aufladung für die hochenergetische Schockabgabe kann ein

\footnotetext{
${ }^{1}$ Patient und andere männliche Formen stehen in dieser Arbeit für geschlechtsneutrale Bezeichnungen.
} 
ATP ausgelöst werden. Dadurch lässt sich oft ein schmerzhafter Schock vermeiden (Schoels et al. 2007). Kann durch ein ATP kein normofrequenter Herzrhythmus hergestellt werden, löst der ICD eine Defibrillation aus (Koneru et al. 2011). Es handelt sich dann um eine sogenannte appropriate Schockabgabe. Bei einer als Kammerflimmern interpretierten Rhythmusstörung oder einer sehr schnellen, hämodynamisch instabilen ventrikulären Tachykardie wird im Allgemeinen primär defibrilliert (Wathen et al. 2001).

\subsection{Studienlage zur präventiven Implantation von ICD-Systemen}

Verschiedene Studien haben den Überlebensvorteil durch eine ICD-Implantation untersucht. Es wird dabei zwischen Patienten, die bereits einen Herz-Kreislauf-Stillstand oder eine hämodynamisch instabile Kammertachykardie überlebt haben (ICD-Implantation zur Sekundärprävention) (Moss 1997; Connolly et al. 2000a; Kuck et al. 2000), und Hochrisikopatienten, welche zum Zeitpunkt der Implantation noch keine lebensbedrohliche Kammertachykardie überlebt haben, unterschieden (Primärprävention) (van Welsenes et al. 2011). In einer Metaanalyse der Studien zur Sekundärprävention konnte unabhängig von der Indexarrhythmie (Kammerflimmern oder hämodynamisch wirksame Kammertachykardie) oder der kardialen Grunderkrankung eine Reduktion der Gesamtmortalität um $28 \%$ bei Patienten beobachtet werden, die neben einer optimierten medikamentösen Therapie zusätzlich mit einem ICD versorgt wurden (Connolly et al. 2000b). Die Ergebnisse der Studien werden in den aktuellen Leitlinien zur Implantation von ICD berücksichtigt (Goldberger et al. 2008, Zipes et al. 2006)

Die SCD-HeFT-Studie (Sudden Cardiac Death in Heart Failure Trial) zeigte bei Patienten mit einer systolischen Herzinsuffizienz (ischämische und nichtischämische Kardiomyopathie; NYHA II-III und linksventrikuläre Ejektionsfraktion (LVEF) $\leq 35 \%$ ), dass signifikant mehr Patienten überlebten, die statt des Antiarrhythmikums Amiodaron im Rahmen einer Primärprävention einen ICD erhielten. Es wurde eine Reduktion der Sterblichkeitsrate um 23 \% durch die Implantation eines ICD gefunden (Bardy et al. 2005). In der MADIT-II-Studie (Multicenter Automatic Defibrillator Implantation Trial II) konnten Patienten im chronischen Postinfarktstadium (LVEF $\leq 30$ \%) einen erheblichen Mortalitätsvorteil durch eine ergänzende primärpräventive Versorgung mit einem ICD erreichen (Moss et al. 2002).

\subsection{Nachteile einer ICD-Implantation}

ICDs definieren eine ventrikuläre Rhythmusstörung, indem die vorliegende Kammerfrequenz mit programmierten Schwellen verglichen wird. Nicht immer handelt es sich bei erhöhter Kammerfrequenz um eine lebensbedrohliche ventrikuläre Arrhythmie. Auch eine supraventrikuläre Tachykardie kann bei schneller Überleitung zu Erhöhung der Kammerfrequenz führen. Da es keine absolut zuverlässigen Algorithmen gibt, um bei gleicher Kammerfrequenz zwischen einer nicht lebensbedrohlichen und einer lebensbedrohlichen Rhythmusstörung zu unterscheiden, ist eine Fehldetektion oder eine Fehlverarbeitung des Signals möglich. Dieses kann zur Auslösung eines nicht notwendigen ATP oder sogar eines Schocks führen (Seegers et al. 2010). Man spricht dann von einer inappropriaten Therapie. 
Hauptursache für eine inappropriate Schockabgabe sind Fehlinterpretationen supraventrikulärer Tachykardien, von denen Vorhofflimmern und Vorhofflattern meistens ursächlich sind (Daubert et al. 2008; Schmitt et al. 1994). Die Abgabe inappropriater ATPs bzw. Schocks kann auch ein Zeichen für eine Fehlfunktion des Aggregats oder des Elektrodensystems wie z. B. ein Sondenisolationsdefekt oder Elektrodenbruch sein (Occhetta et al. 2006; Wollmann et al. 2008). Mit einer Inzidenz von 15 bis $20 \%$ innerhalb von fünf Jahren gehören inappropriate Schockabgaben zu den gefürchteten Komplikationen bei der Versorgung eines Patienten mit einem ICD (van Rees et al. 2011). Häufig sind solche Fehlfunktionen für Patienten sehr schmerzhaft und führen so zu einer Verschlechterung der Lebensqualität (Ladwig et al. 2010; Schron et al. 2002; Sweeney et al. 2005). Göttinger Mediziner veröffentlichten sogar einen Fall, bei dem inappropriate Schocks eine tödliche Arrhythmie induzierten (Vollmann et al. 2005). In der im Jahre 2012 publizierten MADIT-RITStudie (Multicenter Automatic Defibrillator Implantation Trial Reduce Inappropriate Therapy) wurde aufgrund eines möglichen proarrhythmischen Effektes eine Erhöhung der Mortalität durch inappropriate ICD-Therapien beobachtet (Moss et al. 2012).

Weitere negative Aspekte, die mit einer ICD-Implantation verbunden sein können, sind im Folgenden exemplarisch aufgelistet:

- Infektion des ICD-Aggregates (peri- oder postoperativ) (Sohail et al. 2011)

- Bei einigen Patienten ist trotz gegebener Indikation zur ICD-Implantation eine appropriate ICD-Therapie nie erforderlich (Koller et al. 2008).

- Eine Schockabgabe führt anscheinbar zu einer Verschlechterung der Prognose, unabhängig davon, ob sie appropriat oder inappropriat ist (Poole et al. 2008).

- $\quad$ Entstehung von Angststörungen (Benninghoven et al. 2006)

In der AVID-Studie (Antiarrhythmics Versus Implantable Defibrillators), eine der drei großen Studien zur Sekundärprävention, traten bei rund einem Drittel der Patienten innerhalb von zwei Jahren keine anhaltenden Kammertachykardien und kein Kammerflimmern auf (Moss 1997). Sowohl in der MADIT-II-Studie als auch in der SCD-HeFT-Studie erfolgten bei etwa $10 \%$ aller Studienteilnehmer innerhalb von 20 bzw. 45 Monaten inappropriate Therapieabgaben (Germano et al. 2006). Costantini et al. (2009) konnten mit der multizentrischen Studie ABCD (Alternans Before Cardioverter Defibrillator) ergänzend zeigen, dass in einer Patientenpopulation mit MADIT-II-Einschlusskriterien 15 bis 17 ICDs implantiert werden müssen, um ein Patientenleben in einem Zeitraum von zwei Jahren zu retten (sogenannte „Number Needed to Treat“ [NNT]).

In der Zusammenschau verdeutlichen diese Aspekte, dass eine gründliche Risikostratifikation für das Auftreten maligner Herzrhythmusstörungen im Vorfeld einer ICD-Implantation erfolgen sollte, um so eine gute Patientenselektion vornehmen zu können. 


\subsection{Apparative Methoden zur Risikostratifikation}

Aufgrund der in Kapitel 1.4 genannten Komplikationen und Risiken einer ICD-Implantation sollte die Indikation für eine entsprechende Intervention im Rahmen einer individuellen Risikostratifikation genau geprüft werden.

Aktuell stützt sich eine leitlinienkonforme Indikation zur Implantation eines ICD entweder auf ein stattgehabtes kardiales Ereignis (Reanimation, anhaltende VT, arrhythmogene Synkope) oder auf eine kritisch reduzierte LVEF, seltener auch auf invasive Untersuchungen wie eine elektrophysiologische Untersuchung (EPU). Zu den eindeutigen sekundärpräventiven Indikationen gehören eine stattgehabte anhaltende ventrikuläre Tachyarrhythmie oder eine mutmaßlich arrhythmogene Synkope. Darüber hinaus wird die Indikation für eine EPU nur noch selten und eher individuell bei der Evaluation einer leitlinienkonformen Indikation zur Implantation eines ICD gestellt (Willems et al. 2007).

Größere Bedeutung als die invasive EPU erlangen nichtinvasive diagnostische Verfahren. In den großen randomisierten Studien zur ICD-Implantation wird überwiegend die LVEF als Risikoparameter herangezogen (Buxton 1999; Moss 1996; Moss 2002). Auch in den führenden Studien zur primärpräventiven Implantation eines ICD (siehe oben MADIT-II-Studie und SCD-HeFT-Studie) wurden die Patienten durch eine hochgradig reduzierte LVEF identifiziert.

Bereits in der ATRAMI-Studie (Autonomic Tone and Reflexes After Myocardial Infarction) ließ sich die Vermutung bestätigen, dass die LVEF nur eine geringe Sensitivität bietet, um Patienten mit erhöhtem Risiko zu identifizieren (La Rovere et al. 1998).

Bei der Hälfte aller plötzlichen Herztodesfälle betrug die LVEF > 35 \% (La Rovere et al. 2001). Bestätigen konnten dies Barthel et al. (2003), als sie zeigten, dass weniger als $30 \%$ der Patienten, die nach einem Myokardinfarkt sterben, prämortal eine reduzierte LVEF aufwiesen.

Buxton vertritt die Meinung, dass die LVEF als idealer Risikomarker nicht nur eine sehr hohe Sensitivität, sondern auch eine hohe Spezifität haben sollte (Buxton 2005). In seiner Studie MUSTT (Multicenter Unsustained Tachycardia Trial) konnte er der LVEF aber nur eine begrenzte Spezifität zuschreiben (Buxton et al. 2002). Analysen innerhalb der Studie ergaben, dass sich die prognostische Wertigkeit der LVEF in Verbindung mit anderen Risikomarkern wie beispielsweise einer anormalen Baroreflexsensitivität oder in Verbindung mit einer EPU deutlich steigern lässt (Buxton 2005).

Neben der Bestimmung der Baroreflexsensitivität als Zeichen einer autonomen kardialen Regulationsstörung stehen noch weitere nichtinvasive Risikomarker mit unterschiedlichen prädiktiven Werten und klinischem Nutzen zur Verfügung. In der Arrhythmiediagnostik wird zum Erkennen von Erregungsleitungsstörungen die QRS-Breite bei Patienten mit Herzinsuffizienz (Bode-Schnurbus et al. 2003) bestimmt. Untersuchungsverfahren wie das Signalmittlungs-EKG bei Patienten, die einen Herzinfarkt erlitten haben und eine verminderte LVEF aufweisen (Perkiömäki et al. 2011), oder die QRS-Fragmentation mittels Magnetic Field Imaging bei DCM-Patienten (Sha et al. 2011) können als Risikomarker für le- 
bensbedrohliche ventrikuläre Tachyarrhythmien genutzt werden, sind jedoch nicht etabliert. Patienten mit einer verringerten Herzfrequenzvariabilität oder mit Herzfrequenzturbulenzen, die im Langzeit-EKG gemessen werden, scheinen ein erhöhtes Sterberisiko zu haben (Stein und Barzilay 2011). Da Veränderungen der ventrikulären Repolarisation Risikofaktoren für einen SCD darstellen können, werden nichtinvasive Untersuchungsverfahren zur Bestimmung des QT-Intervalls und der QT-Dispersion angewandt (Calò et al. 2011; Okin et al. 2000). Im Folgenden wird die Bedeutung des T-Wellen-Alternans (TWA) beschrieben, der Ausdruck einer ventrikulären Repolarisationsstörung sein kann (Oshodi et al. 2008).

\subsection{T-Wellen-Alternans im Mikrovoltbereich}

In einem Oberflächen-EKG folgt nach dem QRS-Komplex die T-Welle. Sie entspricht dem Ende der intraventrikulären Repolarisation (Schuster und Trappe 2005). Kommt es von Schlag zu Schlag zu einer Veränderung in der Morphologie der T-Welle (Polarität, Form oder Amplitude) ohne Veränderung des RR-Intervalls oder des QRS-Komplexes, wird dieses Phänomen als TWA bezeichnet (Armoundas et al. 2002; Rosenbaum 2001; Schwartz und Malliani 1975; Verrier et al. 2009). Ein TWA tritt typischerweise bei jedem zweiten Schlag in einem ABABAB...-Muster auf (Verrier et al. 2005). Es wird zwischen einem makroskopischen TWA und einem TWA im Mikrovoltbereich (MTWA) unterschieden (Klingenheben et al. 2000). Der MTWA kann aufgrund einer Veränderung im Mikrovoltbereich nur mit Hilfe von computergestützten Diagnosesystemen detektiert werden. Der seit langem bekannte makroskopische TWA ist im Oberflächen-EKG erkennbar (Hering 1909). In Abbildung 1 wird schematisch der Unterschied zwischen einem normalen EKG (oben) und einem EKG mit Amplitudenschwankungen der T-Welle (unten) dargestellt.

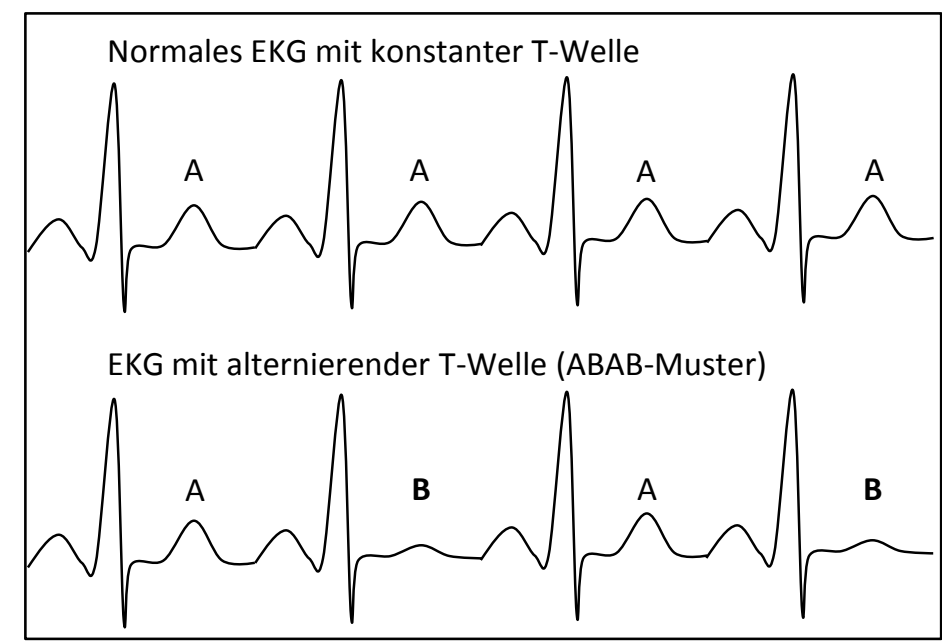

Abb. 1 Schematische Darstellung eines T-Wellen-Alternans. Oben: Normales EKG. Unten: EKG mit alternierender T-Welle im ABAB-Muster. 
Der makroskopische TWA gilt als aussagekräftiger Prädiktor für eine ventrikuläre Tachyarrhythmie, wenn er unter einigen pathologischen Umständen auftritt, wie z. B. bei Patienten mit einem Long-QT-Syndrom (Armoundas et al. 2000; Platt et al. 1996; Schwartz und Malliani 1975). Der MTWA ist ein herzfrequenzabhängiges Phänomen, das sich oberhalb der normalen Ruhefrequenz messen lässt. Die Testung auf das Vorliegen von T-Wellen-Alternans im Mikrovoltbereich (TWA-Testung) kann während einer nichtinvasiven ergometrischen Untersuchung durchgeführt werden (Hohnloser et al. 1998). Anders als bei einer Belastungsergometrie ist bei der TWA-Testung eine submaximale Belastung in der Regel ausreichend (Hohnloser et al. 1997; Myles et al. 2007). Durch experimentelle Arbeiten wird das Auftreten von MTWA auf Repolarisationsstörungen in Myokardzellen zurückgeführt, wobei ein unphysiologischer zellulärer Kalziumstrom am ehesten die Ursache darstellt (Oshodi et al. 2008). Rosenbaum et al. veröffentlichten die erste klinische Studie, in der die prognostische Wertigkeit des MTWA für eine erhöhte Arrhythmieneigung beschrieben wurde (Rosenbaum et al. 1994). Seitdem wird der klinische Nutzen des MTWA für die Risikostratifikation kontrovers diskutiert (Pandit 2010). Die Testung auf das Vorliegen von T-Wellen-Alternans im Mikrovoltbereich (MTWA) ist ein vielversprechendes Untersuchungsverfahren für eine nichtinvasive Risikostratifikation (Cutler und Rosenbaum 2009; Klingenheben et al. 1999; Narayan 2006, Rosenbaum et al. 1996), da das Vorliegen von MTWA nicht nur mit dem Auftreten von ventrikulären Tachyarrhythmien assoziiert ist, sondern auch mit der Gesamtmortalität (Hohnloser 1997; Pastore et al. 1999; Rosenbaum et al. 1994). Entsprechend ist es laut internationalen Leitlinien zur Implantation von Defibrillatoren begründet, eine TWA-Testung durchzuführen, um eine genauere Risikostratifikation für ventrikuläre Arrhythmien zu ermitteln (Evidenzgrad A, Empfehlungsgrad Klasse Ila) (Zipes et al. 2006). Auch wenn die TWA-Testung möglicherweise einen guten Vorhersagewert bietet, hat sie sich bisher als Risikomarker im klinischen Alltag nicht durchsetzen können (Tebbenjohanns et al. 2008). In einer Studie mit einem ähnlichen Kollektiv wie in der MADIT-II-Studie lag die Zwei-Jahres-Mortalität in der Gruppe mit unauffälliger TWA-Testung (3,8\%) wesentlich niedriger als in der Gruppe mit auffälligem Testergebnis (17,8\%) (Bloomfield et al. 2004). Gold et al. (2008) veröffentlichten eine Substudie der SCD-HeFT-Studie, in der das TWA-Ergebnis keinen Vorhersagewert für die Gesamtmortalität besaß. Auch die Endpunkte SCD, anhaltende Kammertachykardie oder appropriate ICD-Therapie wurden in den Gruppen mit auffälliger (nicht-negativ) oder unauffälliger Testung (negativ) nicht signifikant unterschiedlich häufig erreicht.

Die Multizenterstudie $A B C D$ verglich die prognostische Wertigkeit der TWA-Testung und der EPU. Insbesondere wenn beide Testungen durchgeführt wurden, fand man eine Gruppe von Hochrisikopatienten, die von einer ICD-Implantation profitierten. Insgesamt zeigte sich ein sehr hoher negativer prädiktiver Wert ( $\mathrm{npW}$ ) für eine unauffällige bzw. negative TWA-Testung (Bloomfield et al. 2004; Costantini et al. 2009). Hohnloser et al. stellten schon 2003 die Hypothese auf, dass bei präventiver ICD-Indikation und negativem 
Ergebnis in der TWA-Testung auf einen ICD verzichtet werden kann. Jedoch ist bis heute die Aussagekraft der TWA-Testung immer noch nicht hinreichend geklärt. In einer Metaanalyse mit 19 Studien konnte gezeigt werden, dass der MTWA einen signifikanten Vorhersagewert für das Auftreten ventrikulärer Tachyarrhythmien hat (Gehi et al. 2005). In den aktuellen AHA/ACC/ESC-Leitlinien zum Management von Patienten mit ventrikulären Tachyarrhythmien und zur Prävention des plötzlichen Herztodes wird die TWA-Testung derzeit als Risikomarker hinsichtlich lebensbedrohlicher ventrikulärer Arrhythmien mit dem Evidenzgrad $\mathrm{A}^{2}$ und dem Empfehlungsgrad Klasse $\mathrm{Ila}^{3}$ bewertet (Zipes et al. 2006).

\footnotetext{
${ }^{2}$ Evidenzgard A: Daten aus mehreren ausreichend großen, randomisierten Studien oder Metaanalysen.

${ }^{3}$ Empfehlungsgrad Klasse lla: Nutzen/Effektivität einer Maßnahme ist weniger gut durch Evidenzen/Meinungen belegt.
} 


\subsection{Fragestellung und Zielsetzung}

Im Rahmen der vorliegenden Beobachtungsstudie Arbeit soll nach Auswertung routinemäßig durchgeführter TWA-Testungen und einer sich daran anschließenden Nachbeobachtung untersucht werden, ob das Vorliegen einer auffälligen TWA-Testung die Häufigkeit appropriater Schockabgaben und die Gesamtmortalität bei Patienten nach Erstimplantation eines ICD vorhersagen kann. Hierbei erfolgte die Patientenselektion zum einen unabhängig von der kardialen Grunderkrankung, zum anderen unabhängig von der Indikation für die Erstimplantation eines ICD. Auf eine Selektion durch das Maß der LVEF, wie es in vielen anderen Arbeiten üblich war, wurde verzichtet. Bisher wurden wenige Arbeiten zur Risikostratifikation mit einem für den klinischen Alltag repräsentativen Kollektiv veröffentlicht, in der der Stellenwert der TWA-Testung als nichtinvasive Risikostratifikationsmethode für lebensbedrohliche Herzrhythmusstörungen untersucht wurde. Letztlich soll auch die Anwendbarkeit der TWA-Testung im klinischen Alltag überprüft werden. 


\section{Methodik}

\subsection{Patientenkollektiv}

Zwischen Juni 2008 und August 2009 wurde bei 198 Patienten am Herzzentrum der Universitätsmedizin Göttingen (UMG) ein ICD de novo implantiert. Die Indikation zur Implantation wurde anhand aktueller Leitlinien gestellt (Jung et al. 2006; Zipes et al. 2006). Es erfüllten 134 Patienten die Einschlusskriterien für die Durchführung klinisch indizierter Risikostratifikationsmethoden, wobei keine Selektion anhand der kardialen Grunderkrankung oder der Indikation für die ICD-Implantation (Primär- und Sekundärprävention) erfolgte. Die genauen Ein- und Ausschlusskriterien werden in Kapitel 2.2 beschrieben.

In einem ausführlichen Patientengespräch wurden alle Patienten über den Hintergrund und Zweck der TWA-Testung sowie über die Beobachtungsstudie informiert. Nach dem Erhalt eines Patienteninformationsblattes gaben 134 Patienten ihr Einverständnis zur Erfassung ihrer pseudonymisierten Daten aus der routinemäßig durchgeführten TWATestung, bei der es sich um eine zugelassene und empfohlene Risikostratifikationsmethode handelt. Die meisten TWA-Testungen wurden perioperativ und überwiegend vor der Implantation im Rahmen des stationären Aufenthaltes durchgeführt. Konnte keine Testung während des stationären Aufenthaltes erfolgen, wurde der erste postinterventionelle Kontrolltermin in der ICD-Ambulanz der UMG genutzt, um die Testung nachzuholen. Die Patientencharakteristika sind in Kapitel 3.1 dargestellt.

\subsection{Ein- und Ausschlusskriterien}

Das Haupteinschlusskriterium dieser prospektiven Beobachtungsstudie war die geplante Erstimplantation eines ICD. Des Weiteren waren ein Mindestalter von 18 Jahren und das Einverständnis zur Teilnahme erforderlich. Aus methodischen Gründen konnten nur Patienten mit einem der folgenden Herzrhythmen eingeschlossen werden:

- regelmäßiger Sinusrhythmus mit intrinsischer Überleitung auf die Ventrikel,

- durch atriale Stimulation induzierter Vorhofrhythmus mit intrinsischer Überleitung auf die Ventrikel,

- bei atrial getriggerter biventrikulärer Stimulation der Herzhauptkammern durch ein Gerät zur kardialen Resynchronisationstherapie, unabhängig davon, ob ein Sinusrhythmus oder ein durch atriale Stimulation induzierter Vorhofrhythmus vorlag.

Bei Vorliegen eines oder mehrerer der folgenden Ausschlusskriterien wurden Patienten nicht in die Studie aufgenommen:

- Vorhofflimmern oder andere supraventrikuläre Herzrhythmusstörungen, z. B. Vorhofflattern, 
- dauerhafte rechtsventrikuläre Schrittmacherstimulation, die aufgrund eines höhergradigen atrioventrikulären Blockes auch nicht durch ein vorübergehendes Umprogrammieren des ICD vermeidbar war,

- ausschließlich rechtsventrikuläre Stimulation bei einer ICD-gesteuerten TWATestung,

- vor und nach der Implantation Betreuung durch die kinderkardiologische Abteilung,

- kardiale Dekompensation oder akute Myokardischämie.

\subsection{T-Wellen-Alternans-Testung}

Der MTWA ist ein herzfrequenzabhängiges Phänomen, welches eher außerhalb der Ruhefrequenz auftritt. Daher ist für eine TWA-Testung eine moderate Herzfrequenzanhebung notwendig. Mit Hilfe von Spezialelektroden zur Artefaktreduzierung und Computertechnologie nebst speziell entwickelter Software ist es möglich, während einer Ergometrie nichtinvasiv eine TWA-Testung durchzuführen (Bloomfield 2002). Ein Herzfrequenzbereich von 100 bis 120 Schlägen/min ist für eine Testung optimal (Kavesh et al. 1998).

\subsubsection{Material und Vorbereitungen für eine TWA-Testung}

Alle TWA-Testungen wurden mit dem Diagnosesystem $\mathrm{CH}_{2000}{ }^{\mathrm{TM}}$ der Firma Cambridge Heart (Cambridge Heart, Inc. Bedford, MA, USA) durchgeführt. Das System basiert auf einem IBM-kompatiblen Personal Computer (IBM Corporation, Armonk, USA) mit dem Betriebssystem Windows $2000^{\mathrm{TM}}$ von Microsoft Corporation (Microsoft Corporation, Redmond, USA). Mit der dazugehörigen Software (Version 3.1.1) und einer spektralanalytischen Berechnung ist es möglich, MTWA zu detektieren (Smith et al. 1988).

Bei einer ergometrisch geführten TWA-Testung wurde das Sitzergometer ergoselect der Firma ergoline (ergoline $\mathrm{GmbH}$, Bitz, Deutschland) verwendet, das über ein serielles Datenkommunikationskabel mit einer EIA-232-Schnittstelle an das $\mathrm{CH}_{2000}{ }^{\mathrm{TM}}$ gekoppelt war. Dies ermöglichte eine individuelle Steuerung der Belastung per Tastatur des $\mathrm{CH} 2000^{\mathrm{TM}}$ und eine Kontrolle der Radumdrehungen pro Minute.

Eine aussagekräftige TWA-Testung erfordert eine gute Signalqualität mit einem guten Signal-Rausch-Verhältnis zugunsten des Signals. Dazu erfolgte eine gründliche Hautpräparation, die bei Körperbehaarung eine Rasur mittels Einmalrasierer beinhaltete. Zur Verringerung des Hautwiderstands und zur Erhöhung der Signalqualität wurde vor dem Kleben der Elektroden die Epidermis mit dem medizinischen Schleifpapier One Step Skin Prep $^{\mathrm{TM}}$ der Firma 3M (3M AG, Rüschlikon, Schweiz) oberflächlich und vorsichtig angeraut. Unmittelbar nach dem Anrauen der Haut wurden die Elektroden positioniert, um ein Kleben an einer unpräparierten Stelle zu vermeiden. Die Elektroden wurden in der in Abbildung 2 gezeigten Konfiguration positioniert. Neben sieben handelsüblichen Silber/Silberchlorid-Elektroden der Firma Ambu (Ambu GmbH, Bad Nauheim, Deutschland), entsprechend den runden Ableitungspunkten, kamen ergänzend Multikontaktelektroden 
(Micro-V Alternans Sensor ${ }^{\mathrm{TM}}$, Cambridge Heart, Inc. Bedford, MA, USA) an den mit Quadraten gekennzeichneten Ableitungspunkten zum Einsatz (siehe Abbildungen 2 und 3).

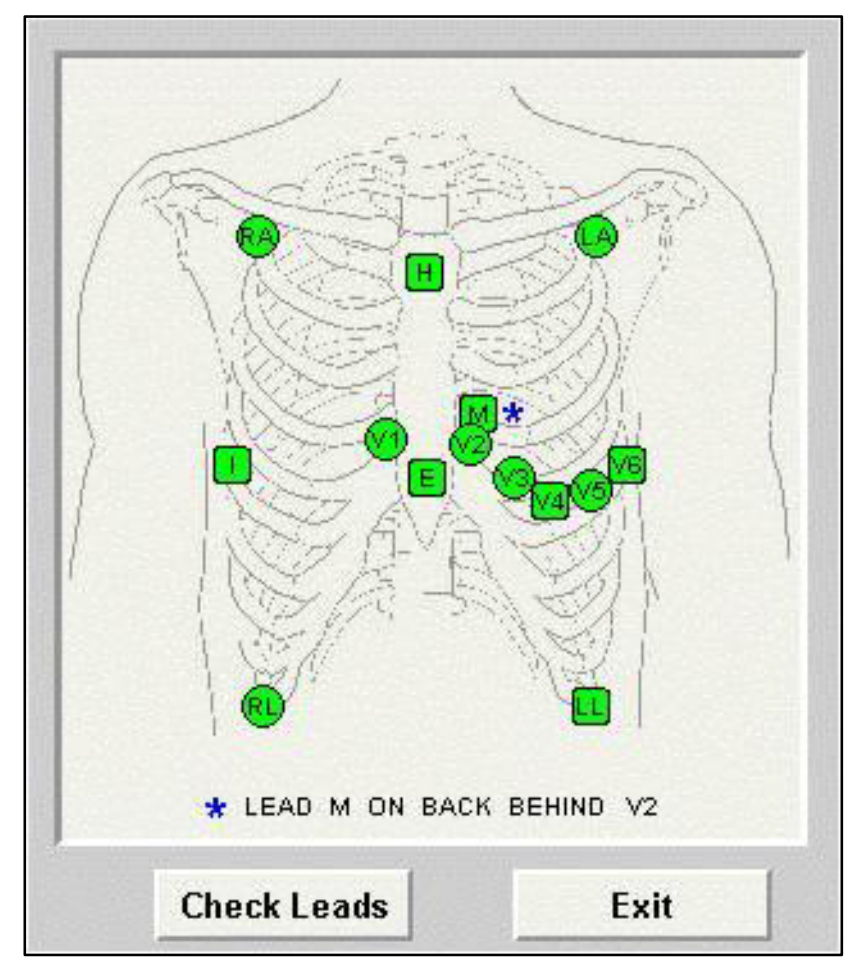

Abb. 2 Elektrodenkonfiguration aller 14 Elektroden (Screenshot von Software des $\mathrm{CH}_{2000^{T M}}$ Version 3.1.1). Quadratisches Symbol entspricht einer Multikontaktelektrode. Silber-/Silberchlorid-Elektroden werden durch runde Symbole dargestellt (siehe auch Kapitel 6.1). Bei diesem Beispiel wird an allen Elektroden eine gute Konnektivität gemessen (grüne Symbole). Mit freundlicher Genehmigung von Cambridge Heart, Inc.

Neben einem 12-Kanal-EKG wurden zusätzlich bipolare Ableitungen nach Frank im Rahmen einer Vektorkardiografie aufgezeichnet (orthogonale Achsen $X, Y$ und Z), aus denen die vektorielle Magnitudenableitung (VM) abgeleitet wurde (Frank 1956). Eine detaillierte Beschreibung der Elektrodenposition befindet sich in Kapitel 6.1. Nach dem Kleben der Elektroden kann der Widerstand als ein Maß für die Konnektivität am Monitor des $\mathrm{CH}_{2000}{ }^{\mathrm{TM}}$ beurteilt werden. Für eine erfolgreiche Messung ist ein Widerstand von weniger als $3 \mathrm{k} \Omega$ notwendig. Die Rückmeldung des $\mathrm{CH}_{2000}{ }^{\mathrm{TM}}$ über den Widerstand erfolgt mittels Ampelprinzip. Grüne Symbole signalisieren eine gute und gelbe Symbole eine ausreichende Konnektivität. Bei Rot wurde durch eine zusätzliche Hautpräparation versucht, eine bessere Konnektivität zu erzielen (siehe Abbildung 2).

Die in Abbildung 3 gezeigte Multikontaktelektrode kann ein Verfälschen des Frequenzspektrums durch Muskelartefakt-bedingte Störsignale (Bewegung und Atmung) 
verringern. Die Elektrode besteht aus einem runden zentralen Segment und drei halbkreisförmigen Segmenten, die das Mittelsegment umgeben. Über vier getrennte Aufnahmefelder können um einen anatomischen Punkt die gemessenen Potentiale gemittelt werden, um störende Artefakte zu minimieren (Albrecht et al. 1996).

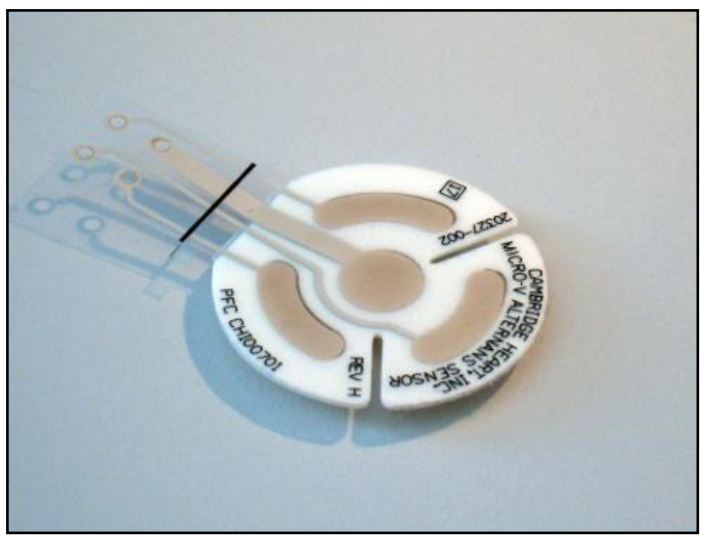

Abb. 3 Multikontaktelektrode (Micro-V Alternans Sensor ${ }^{\mathrm{TM}}$ der Firma Cambridge Heart, Inc.) 
Die EKG-Signale der Elektroden wurden über 14 Ableitungen zu einem Patientenmodul namens PM- $3^{\mathrm{TM}}$ der Firma Cambridge Heart, Inc. geleitet, das der Patient an einem Gürtel während der Testung trug (siehe Abbildung 4). Innerhalb des Moduls erfolgte die Digitalisierung der Signale mittels 22-Bit-Analog-Digitalwandlers. Die digitalisierten Signale wurden potentialgetrennt über einen Optokoppler übertragen und per Kabel seriell an den Personal Computer des $\mathrm{CH} 2000^{\mathrm{TM}}$ gesendet. Das Patientenmodul wurde über einen kleinen Transformator potentialgetrennt mit Energie versorgt.

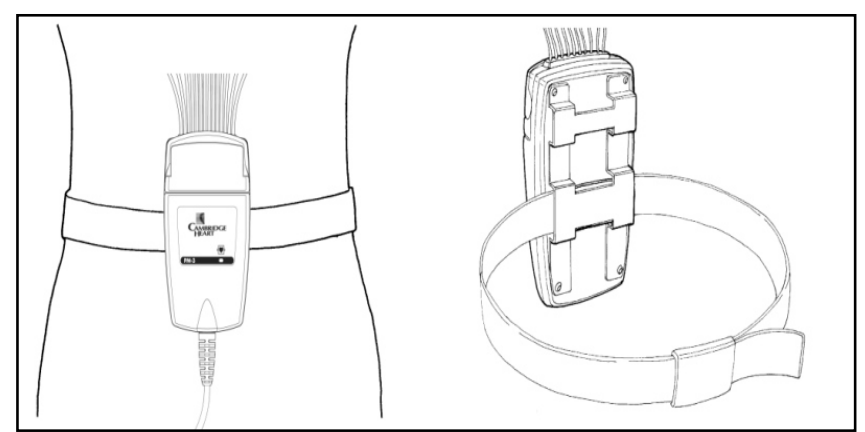

Abb. 4 Patientenmodul PM-3 $3^{T M}$ der Firma Cambridge Heart, Inc. Mit freundlicher Genehmigung von Cambridge Heart, Inc.

Jede fahrradergometrische TWA-Testung wurde in Anlehnung an die Empfehlungen zur Durchführung einer klassischen Ergometrie durchgeführt. Sofern eine TWA-Testung eine temporäre Änderung der Stimulation erforderte, war das empfohlene Equipment zur Nachsorge vorhanden. Alle TWA-Testungen wurden von einem qualifizierten Arzt des Herzzentrums der UMG begleitet.

\subsubsection{Ablauf einer TWA-Testung}

Nach einer einminütigen Messung in Ruhe erfolgte die erste Belastungsphase. Ausgehend von einer anfänglichen Belastung von 20 Watt wurde der zu überwindende Widerstand so angepasst, dass die Herzfrequenz einen steady state im Bereich von 100 bis 110 Schlägen/min erreichte. Es wurde auf eine langsame Leistungssteigerung geachtet, da ein schneller Herzfrequenzanstieg ein falsch positives Testergebnis provozieren kann (Cutler und Rosenbaum 2009). Im ersten Bereich sollte die Frequenz 2,5 Minuten gehalten werden, bis das Diagnosesystem $\mathrm{CH}_{2000}{ }^{\mathrm{TM}}$ eine ausreichende Anzahl an Herzschlägen für die Analyse registriert hatte. Direkt daran anschließend erfolgte eine zweite Belastungsphase, in der die Herzfrequenz durch individuelle Anpassung der Leistung auf 110 bis 120 Schläge/min gesteigert wurde. Diese Phase dauerte 1,5 Minuten. Nach der zweiten Belastungsphase wurde die TWA-Testung beendet. Die Belastung wurde langsam reduziert, bis die Herzfrequenz der Ruhefrequenz entsprach. In beiden Phasen wurde versucht, durch eine individuelle Belastung Herzfrequenzschwankungen zu vermeiden, da diese zu störenden Artefakten führen können. 
Zur Verringerung der bewegungsbedingten Artefakte wurde der Patient angehalten, die Arme entspannt herabhängen zu lassen und den Oberkörper wenig zu bewegen, sofern dies für den Patienten möglich war. Weiterhin wurde dem Patienten eine Trittfrequenz vorgegeben, die $33 \%$ oder $66 \%$ der aktuellen Herzfrequenz betrug, da es in einem Bereich von $50 \%$ der Herzfrequenz bei der hier angewandten Spektralanalyse zu einem falsch positiven Testergebnis kommen kann (Hohnloser et al. 1997).

War eine fahrradergometrische Belastung nicht möglich, wurde die TWA-Testung nach ICD-Implantation durch eine temporäre Frequenzanhebung mittels Schrittmacherfunktion des ICD und entsprechenden ICD-Programmiergerätes durchgeführt, sofern ein Gerät mit atrialer Sonde oder ein biventrikuläres Gerät implantiert wurde. Vor der Entfernung der Elektroden vom Oberkörper des Patienten analysierte das $\mathrm{CH}_{2000}{ }^{\mathrm{TM}}$ die gewonnen Daten. Im Anschluss daran wurde zur Interpretation der TWA-Testung ein TWA-Bericht ausgedruckt. Abbildung 5 zeigt beispielhaft einen TWA-Bericht mit einem positiven Testergebnis.

\subsection{Interpretation einer TWA-Testung}

Die Interpretation der TWA-Berichte wurde von zwei voneinander unabhängigen und erfahrenen Ärzten aus der Abteilung Elektrophysiologie des Herzzentrums Göttingen mit Hilfe standardisierter Interpretationsrichtlinien von Bloomfield et al. (2002) vorgenommen.

Mit Hilfe der Richtlinien können die TWA-Testungen folgendermaßen klassifiziert werden:

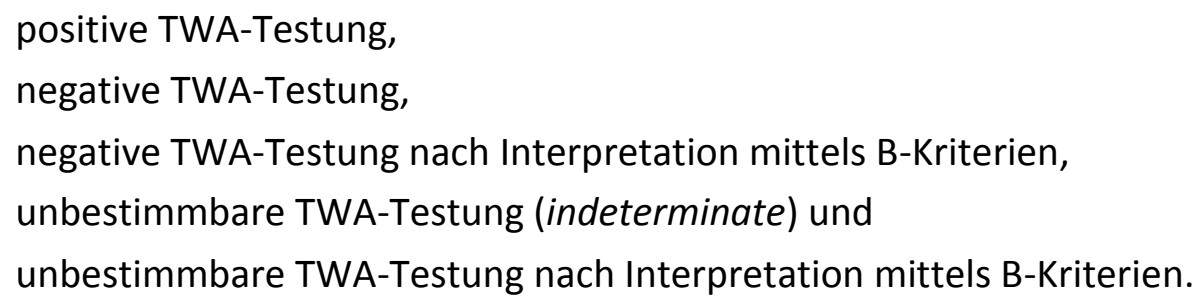

Grundlegend für die Interpretation einer TWA-Testung ist die Frage nach dem Vorliegen eines Daueralternans (sustained alternans), sowie die Bestimmung der patientenspezifischen Alternansschwelle („Onset-Herzfrequenz“). Des Weiteren sind die Bestimmung der maximalen negativen Herzfrequenz (maxNegHR), der maximal erreichten Herzfrequenz und der sogenannten smoothed heart rate wichtig (Richter et al. 2005). Im Folgenden werden diese Parameter erläutert.

\section{Geglättete Herzfrequenz (smoothed heart rate)}

Die sogenannte smoothed heart rate, was mit "geglättete Herzfrequenz" übersetzt werden kann, ist die durchschnittliche Herzfrequenz, die über ein Intervall von 128 Herzschlägen gemittelt wurde und auf den angezeigten Zeitpunkt (64 Schläge vorher und 64 
Schläge nachher) zentriert ist. Im TWA-Bericht wird sie als durchgezogene Linie im Feld „Herzfrequenz" (HF) angezeigt.

\section{Maximale negative Herzfrequenz}

Die maximale negative Herzfrequenz (maxNegHR) beschreibt das einminütige Intervall mit der höchsten smoothed heart rate, in dem kein signifikantes MTWA vorliegt und gleichzeitig Störsignale in der vektorverstärkten Ableitung VM (vector magnitude) $\leq 1,8 \mu \mathrm{V}$ und die ektopen Schläge $\leq 10 \%$ betragen. Darüber hinaus darf keine Ableitungsfehlfunktion bestehen. Sofern kein solches Intervall besteht, wird der Wert definitionsgemäß auf null gesetzt. Ist in dem Intervall die maximale Herzfrequenz enthalten, entspricht die maxNegHR der maximalen Herzfrequenz, ansonsten wird die maxNegHR aus der smoothed heart rate zu Beginn des einminütigen Intervalles ermittelt. Wird Daueralternans festgestellt (siehe unten), entspricht die MaxNegHR der "Onset-Herzfrequenz", wenn zuvor das einminütige Intervall die Kriterien für die MaxNegHR erfüllt und nicht der smoothed heart rate zu Beginn des einminütigen Intervalles.

\section{Maximale Herzfrequenz}

Die maximale Herzfrequenz entspricht der höchsten smoothed heart rate.

\section{Patientenspezifische Alternansschwelle („Onset-Herzfrequenz")}

Die patientenspezifische Alternansschwelle, "Onset-Herzfrequenz" genannt, ist die niedrigste smoothed heart rate, oberhalb derer ein Daueralternans lückenlos auftritt. Ausnahmen bilden Lücken, die durch Störsignale, Herzfrequenzabfall oder durch ektope Herzschläge bedingt sind.

\section{Signifikanter MTWA und Daueralternans (sustained alternans)}

Der signifikante MTWA ist definiert als Alternans, der mit einer Alternansspannung $V_{\text {alt }} \geq 1,9 \mu \mathrm{V}$ und einem Alternansquotienten (K-Wert) $\geq 3$ (im TWA-Bericht als grau schattierter Bereich unter der Kurve markiert) in mindestens einer Ableitung VM, X, Y oder Z oder in zwei zusammenhängenden Brustwandableitungen $V_{1}-V_{6}$ auftritt. Von Daueralternans wird gesprochen, wenn ein signifikanter MTWA lückenlos oberhalb der „OnsetHerzfrequenz" mit einer Dauer von mindesten einer Minute artefaktfrei auftritt (Bloomfield et al. 2002; Hohnloser 2004).

Eine artefaktfreie Aufzeichnung ist durch folgende Kriterien definiert:

Der Anteil an ektopen und vorzeitigen Herzschlägen (z. B. supraventrikuläre und ventrikuläre Extrasystolen) liegt unter 10 \% (in dem TWA-Bericht im Feld „\% schlecht"). 
- $\quad$ Die Trittfrequenz entspricht etwa 1/3 oder 2/3 der Herzfrequenz (im TWABericht im Feld „U/min“).

- Die Atemfrequenz sollte im Gesamten 25 \% der Herzfrequenz (0,25 Zyklen pro Herzschlag) betragen (im TWA-Bericht im Feld „Reaktion“).

- $\quad$ Der R-R-Intervall-Alternans ist $\geq 2$ ms bei einem Alternansquotienten von $\geq 3$ (im TWA-Bericht im Feld „RR Alternans“).

- $\quad$ Die Variation der Herzfrequenz über 128 Herzschlägen beträgt < 30 Schläge/min (im TWA-Bericht im Feld „HF Delta“).

In einem TWA-Bericht signalisiert ein schwarzer Balken unter der Fläche eine artefaktfreie Messperiode (siehe Abbildung 5).

\subsubsection{Positive TWA-Testung}

Eine positive TWA-Testung liegt vor, wenn die Kriterien für einen Daueralternans bei einer "Onset-Herzfrequenz" von $\leq 110$ Schlägen/min erfüllt sind. Da es auch bei Personen ohne erhöhtes Risiko für maligne Herzrhythmusstörungen bei höheren Herzfrequenzen zur Entwicklung eines Daueralternans kommen kann, wird eine "Onset-Herzfrequenz" von $\leq 110$ Schlägen/min gewählt. Gelegentlich wird MTWA in Ruhe beobachtet. In diesem Fall ist der Test unabhängig von der „Onset-Herzfrequenz" als positiv zu werten.

\subsubsection{Negative TWA-Testung}

Ein Testergebnis wird als „negativ“ eingestuft, wenn die Kriterien für einen Daueralternans während der gesamten Testung nicht erfüllt sind. Auch bei einer maximalen negativen Herzfrequenz von $\geq 105$ Schlägen/min resultiert eine negative Testbewertung.

\subsubsection{Unbestimmbare TWA-Testung}

Eine Testung wird als „unbestimmbar" (indeterminate) klassifiziert, wenn die Kriterien weder für eine positive noch für eine negative Testung erfüllt sind.

Häufige Ursachen für unbestimmbare TWA-Testungen sind hier aufgeführt:

Es wird keine maximale Herzfrequenz $\geq 80$ Schlägen/min erreicht.

Der Anteil an Extrasystolen an der Anzahl der Gesamtschläge überschreitet $10 \%$.

- $\quad$ Die Störsignale sind zu hoch.

- $\quad$ Zu große Herzfrequenzschwankungen oder atmungsbedingte Artefakte lassen keine sichere Klassifizierung in „positiv“ oder „negativ“ zu.

\subsubsection{Negative TWA-Testung nach Interpretation mittels B-Kriterien}

Die B-Kriterien erlauben eine weitere Differenzierung von unbestimmbaren Testungen, wenn die maximal erreichte Herzfrequenz von mindestens 80 Schlägen/min erreicht und die ergometrische Belastung beispielsweise durch Ermüdung beendet wird. Beträgt die 
Differenz aus maximal erreichter Herzfrequenz und maximaler negativer Herzfrequenz $\leq 5$ Schläge/min, kann die TWA-Testung als „negativ“ gewertet werden.

\subsubsection{Unbestimmbare TWA-Testung nach Interpretation mittels B-Kriterien}

Wenn lediglich eine maximale Herzfrequenz unterhalb von 80 Schlägen/min ohne Daueralternans erreicht wird, resultiert ein unbestimmbares Testergebnis nach B-Kriterien. Steigt die Herzfrequenz auf mindestens 80 Schläge/min und die Differenz aus maximal erreichter Herzfrequenz und maximaler negativer Herzfrequenz beträgt $\geq 5$ Schläge/min, so lautet das Ergebnis der TWA-Testung „unbestimmbar“ (vgl. 2.4.3).

\subsubsection{Nicht-negative TWA-Testung}

Unter dem Ergebnis „nicht-negativ“ werden alle Testungen geführt, die entweder „unbestimmbar" oder „positiv“ zu werten sind. Durch diese Zusammenfassung gelingt eine dichotome Auswertung von auffälligen („nicht-negativ“) gegenüber unauffälligen Testungen („negativ“) (Bloomfield et al. 2006; Chow et al. 2006; Chow et al. 2007; Gehi et al. 2005; Kaufman et al. 2006). 


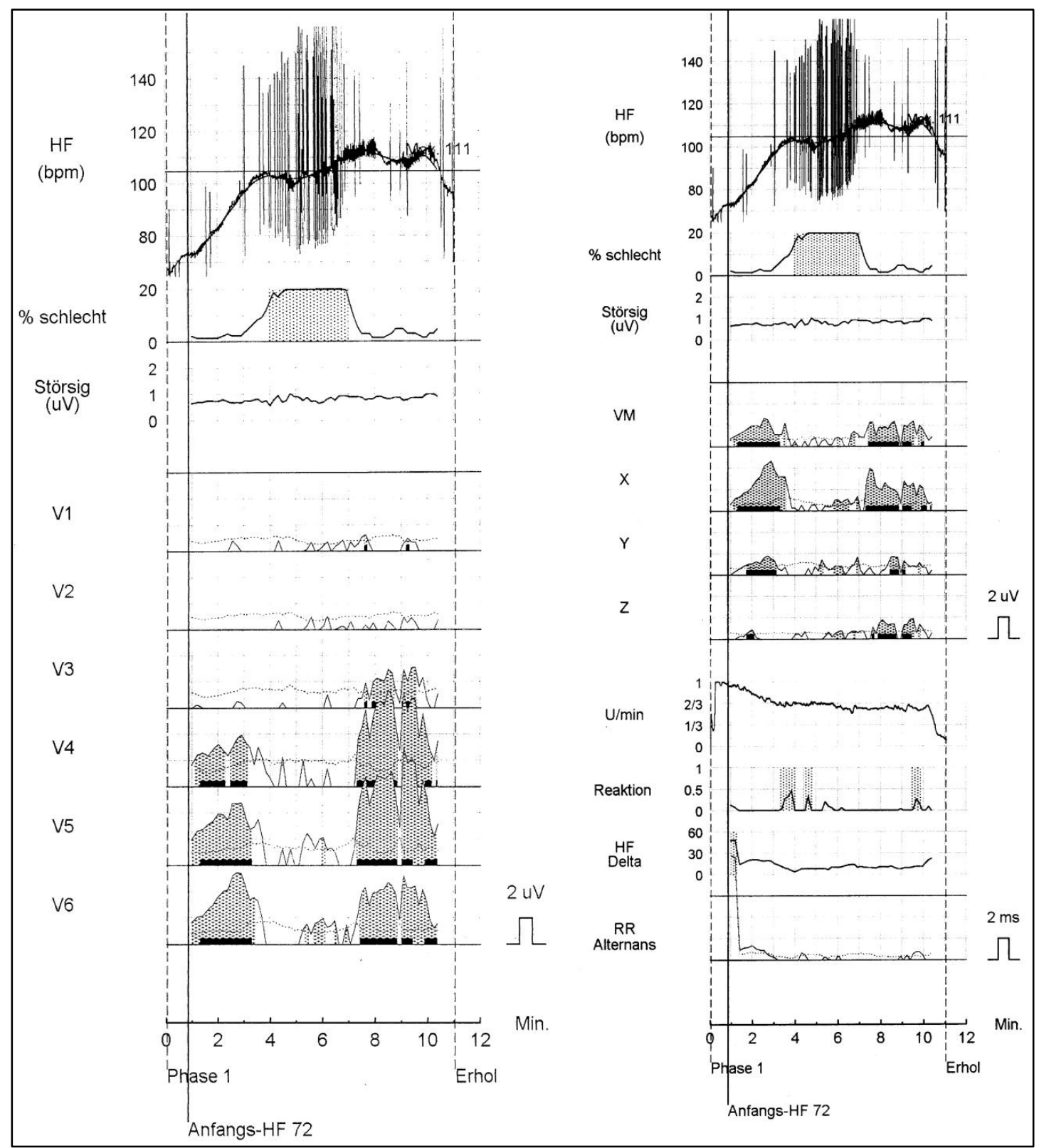

Abb. 5 Beispiel eines TWA-Berichtes von einem 69-jährigen Patienten mit hypertensiver Herzerkrankung im Stadium NYHA II und einer LVEF von $30 \%$. Man erkennt deutlich die Entwicklung eines signifikanten MTWA in den Ableitungen V4-V6, VM und X ab einer Durchschnittsfrequenz von 72 Schlägen/min. Da die Unterbrechung zwischen Minute vier und sieben durch eine Phase von vermehrten ektopen Schlägen erklärt ist (siehe Felder „HF“ und „\% schlecht“), liegt hier Daueralternans oberhalb der „Onset-Frequenz" vor. Das Testergebnis lautet somit „positiv“. 


\subsection{Endpunktdefinition}

Vor Beginn der Datenerhebung wurden „Gesamtmortalität“ und „erste appropriate ICDSchockabgabe“, bedingt durch eine ventrikuläre Tachyarrhythmie, als Endpunkte definiert. Zudem wurden sie kombiniert betrachtet.

\subsection{Nachbeobachtungsphase}

Die Nachbeobachtungszeit endete im Juni 2010 und betrug im Mittel 12 Monate. Im Rahmen der Routinekontrollen in der ICD-Ambulanz des Herzzentrums der UMG wurden appropriate Schockabgaben in einem zeitlichen Abstand von etwa drei bis sechs Monaten durch Auslesung der ICD-Daten als relevantes Ereignis dokumentiert. War dies nicht möglich, wurden die ICD-Daten in Kooperation mit dem Hausarzt und dem Kardiologen erfragt. Die Mortalität wurde durch Hinweise von Angehörigen, des Hausarztes und durch Arztbriefe ermittelt.

\subsection{Statistische Analyse}

Die erhobenen Patientendaten wurden auf einem singulären Computer verwaltet. Es wurde keine Bearbeitung und Speicherung außerhalb dieses Bereiches vorgenommen. Die statistische Analyse erfolgte mit der Software SPSS Statistics ${ }^{T M}$ Version 19 der Firma IBM Corporation. Die Dauer des ereignisfreien Überlebens in Abhängigkeit des TWA-Befundes („negativ“ vs. „,nicht-negativ“ bzw. „,negativ“ vs. „unbestimmbar“ vs. „positiv“; siehe Kapitel 2.4.1 - 2.4.6 und 4.4) wurde mit Hilfe der Kaplan-Meier-Methode analysiert und mittels des Log-Rank-Tests auf signifikante Unterschiede zwischen den Gruppen untersucht. Graphiken wurden mit dem Programm SigmaPlot ${ }^{\mathrm{TM}}$ Version 12 der Firma Systat Software $\mathrm{GmbH}$ erstellt. Säulen- und Tortendiagramme wurden mit Hilfe von Excel $2007^{\mathrm{TM}}$ der Firma Microsoft Corporation gezeichnet.

Die univariate Vorhersage des Risikos eines Ereignisses durch die TWA-Testung wurde durch zweiseitige exakte Fisher-Tests bestimmt, da einige Einzelhäufigkeiten kleiner als fünf betrugen. Eine Irrtumswahrscheinlichkeit von unter $5 \%(p \leq 0,05)$ wurde als statistisch signifikant definiert. Der negative prädiktive Wert wurde als $\frac{\text { Anzahl der richtig negativen Testergebnisse }}{\text { (Anzahl der richtig negativen Testergebnisse +Anzahl der falsch negativen Testergebnisse) }}$ definiert. 


\section{Ergebnisse}

\subsection{Patientencharakteristika}

In die prospektiv durchgeführte Studie wurden 134 Patienten eingeschlossen. Darunter befanden sich 28 Frauen (21\%) und 106 Männer (79\%) mit einem Durchschnittsalter von $64 \pm 13$ Jahren. Die mittlere linksventrikuläre Ejektionsfraktion (LVEF) der Patienten betrug $31 \pm 12 \%$, wobei $78 \%$ eine LVEF $\leq 35 \%$ hatten. Die beiden häufigsten zugrundeliegenden Herzerkrankungen waren bei 72 Patienten ( $54 \%$ ) eine ischämische Kardiomyopathie und bei 35 Patienten (26\%) eine dilatative Kardiomyopathie (vgl. Tabelle 1). Der ICD wurde bei 88 Patienten (66 \%) aus primärpräventiver Indikation implantiert, die übrigen Implantationen erfolgten zur Sekundärprävention. Bei 34 Patienten wurde statt oder in Ergänzung der Ergometrie zur Messung des MTWA eine temporäre Frequenzanhebung mittels ICD durchgeführt. Abhängig vom implantierten ICD-Typ wurde diese bei 18 Patienten durch eine atriale Stimulation und bei 16 durch eine biventrikuläre Stimulation vorgenommen.

\begin{tabular}{|c|c|}
\hline \multicolumn{2}{|c|}{ Tabelle 1} \\
\hline Patientenanzahl (n) & 134 \\
\hline Männlich & $106(79 \%)$ \\
\hline Weiblich & 28 (21 \%) \\
\hline Alter (Jahre) & 64 (range 19-89) \\
\hline \multicolumn{2}{|l|}{ Zugrundeliegende Herzerkrankung } \\
\hline Ischämische Kardiomyopathie & $72(54 \%)$ \\
\hline Dilatative Kardiomyopathie & $35(26 \%)$ \\
\hline Stattgehabter Myokardinfarkt & $21(15 \%)$ \\
\hline Hypertensive Herzerkrankung & $1(1 \%)$ \\
\hline $\begin{array}{l}\text { Arrhythmogene rechtsventrikuläre Kar- } \\
\text { diomyopathie }\end{array}$ & $2(2 \%)$ \\
\hline Hypertrophe Kardiomyopathie & $3(2 \%)$ \\
\hline \multicolumn{2}{|l|}{ ICD-Indikation } \\
\hline Primärpräventive Indikation & $88(66 \%)$ \\
\hline Sekundärpräventive Indikation & $46(34 \%)$ \\
\hline \multicolumn{2}{|l|}{ Ejektionsfraktion } \\
\hline Durchschnittliche linksventrikuläre & $31 \pm 12 \%$ (range $10-60)$ \\
\hline \multicolumn{2}{|l|}{ Ejektionsfraktion } \\
\hline$\leq 35 \%$ & $104(78 \%)$ \\
\hline$>35 \%$ & $30(22 \%)$ \\
\hline \multicolumn{2}{|l|}{ Belastung } \\
\hline Fahrradergometrie & $100(75 \%)$ \\
\hline Pacing mittels ICD-Programmiergerät & $34(25 \%)$ \\
\hline \multicolumn{2}{|l|}{ Durchschnittliche Nachbeobachtungszeit } \\
\hline (Tage) & $368 \pm 160$ \\
\hline
\end{tabular}


Wie in Kapitel 2.1 beschrieben, konnten von den 198 Patienten mit einer ICD-Erstimplantation nur 134 Patienten in die prospektive Studie aufgenommen werden. Die Tabelle 2 gibt einen Überblick darüber, aus welchem Grund bei den übrigen 64 Patienten keine TWA-Testung durchgeführt wurde.

\begin{tabular}{lc}
\multicolumn{1}{c}{ Tabelle 2 } \\
\multicolumn{1}{c}{ Patienten mit Erstimplantation ohne TWA-Testung } \\
\hline Patientenanzahl (n) & 64 \\
Kein Sinusrhythmus & $39(61 \%)$ \\
Keine Ergometrie möglich und rechtsventrikuläre & $8(12 \%)$ \\
$\quad$ Stimulation (1-Kammer-ICD) & \\
Kein Einverständnis & $5(8 \%)$ \\
Kontraindikation für eine Herzfrequenz- oder & $5(8 \%)$ \\
$\quad$ Blutdruckanhebung, bespielsweise bei bekanntem & \\
$\quad$ Aneurysma & $7(11 \%)$ \\
\hline Sonstige Gründe
\end{tabular}

\subsection{Ergebnisse der TWA-Testungen}

Abbildung 6 zeigt die prozentuale Verteilung der Ergebnisse aller 134 TWA-Testungen. Jede Testung wurde mit Hilfe der Interpretationsrichtlinien von Bloomfield et al. (2002) interpretiert (vgl. Kapitel 2.4). 69 Testungen (51\%) erfüllten die Kriterien für ein negatives Ergebnis. 20 der 69 negativen Ergebnisse wurden mittels B-Kriterien ermittelt (vgl. Kapitel 2.4.3). Des Weiteren wiesen 50 Patienten (37\%) ein positives Testergebnis auf. Bei 15 Patienten (11\%) wurde die Testung als unbestimmbar gewertet, wobei meist eine unzureichende maximale Herzfrequenz ( $53 \%$ ) oder ein zu hoher prozentualer Anteil an Extrasystolen (27\%) die Ursache darstellen (siehe Tabelle 3).

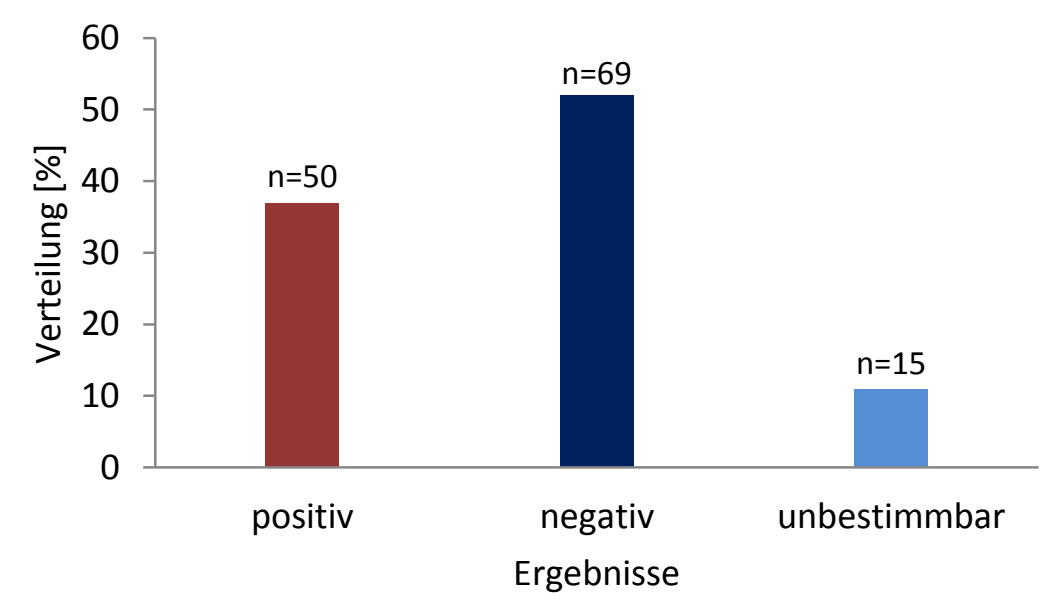

Abb. 6 Prozentuale Verteilung der Ergebnisse aller 134 TWA-Testungen 
Aufgrund vorangegangener Studien wurden zur besseren Vergleichbarkeit positive und unbestimmbare TWA-Testungen zur sogenannten „nicht-negativen“ Gruppe zusammengefasst. Die Verteilung der 69 negativen gegenüber den 65 nicht-negativen Testergebnissen ist in Abbildung 7 dargestellt.

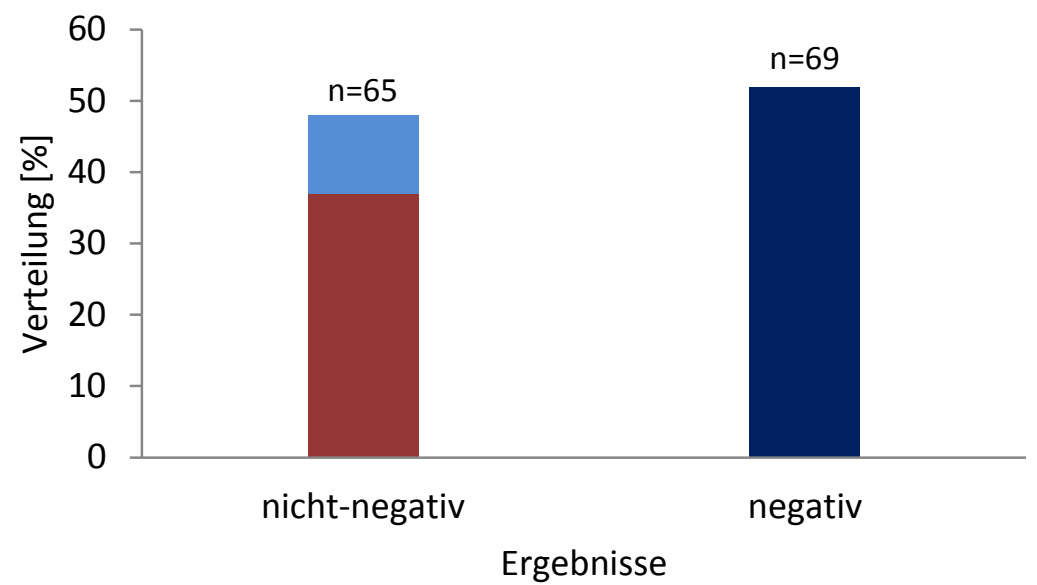

Abb. 7 Verteilung der nicht-negativen gegenüber den negativen Testergebnissen

\subsection{Ursachen für unbestimmbare TWA-Testungen}

Wie in den Kapiteln 2.4.3 und 2.4.5 beschrieben, können verschiedene Umstände während der TWA-Testung zu einem unbestimmbaren Testergebnis führen. Die Tabelle 3 zeigt die Verteilung der unterschiedlichen Ursachen für die insgesamt 15 unbestimmbaren TWA-Testungen. Am häufigsten waren eine unzureichende maximale Herzfrequenz (53 \%) und ein zu hoher Anteil ektoper Herzschläge (27\%) verantwortlich.

\section{Tabelle 3}

Ursachen für unbestimmbare TWA-Testungen

Anzahl unbestimmbarer TWA-Testungen ( $\mathrm{n}$ ) 15

Unzureichende maximale Herzfrequenz

z. B. aufgrund körperlicher Erschöpfung

Ein zu hoher Anteil ektoper Herzschläge

(ventrikuläre und supraventrikuläre Extrasystolen)

Schlechte Signalqualität

z. B. aufgrund verfälschender Störsignale

Andere Gründe $1(7 \%)$ 


\subsection{Nachbeobachtung}

Die mittlere Nachbeobachtungszeit betrug 12,1 \pm 5,2 Monate. Der kürzeste Nachbeobachtungszeitraum bis zum Erreichen eines definierten Endpunktes lag bei 14 Tagen, der längste bei 16,4 Monaten. Bei zwei Patienten war es nicht möglich, innerhalb des Beobachtungszeitraums Informationen über die weitere Krankengeschichte zu erhalten, daher konnten die Ausgangsdaten dieser Patienten bei der statistischen Auswertung nicht berücksichtigt werden. Die Testergebnisse dieser Patienten waren positiv. In der statistischen Analyse konnten folglich nur 132 der 134 Patienten berücksichtigt werden.

\subsection{Ereignisse während der Nachbeobachtungszeit}

Während der Nachbeobachtungszeit traten insgesamt elf Ereignisse auf, die als primärer Endpunkt vordefiniert waren (siehe Abbildung 8). Insgesamt starben acht Patienten. Zweimal war eine kardiale Ursache verantwortlich. Diese Patienten befanden sich in der Gruppe mit negativer TWA-Testung. Zu einer kardialen Ursache zählten ein tödlich verlaufender Myokardinfarkt (Eintritt des Todes innerhalb von 28 Tagen nach Myokardinfarkt), SCD, Tod aufgrund einer Herzinsuffizienz und Tod infolge einer KHK während oder innerhalb 28 Tagen nach einer Intervention sowie alle anderen Todesursachen, die durch eine KHK bedingt sind. Bei den übrigen sechs Patienten lag keine kardiale Ursache vor oder die Todesursache konnte nicht eruiert werden. Die ermittelte jährliche Todesrate betrug 6,1 \% Todesfälle pro Jahr.

Eine appropriate Schockabgabe wurde bei drei Patienten registriert. Die einzelnen Schockabgaben ereigneten sich am 74., 100. bzw. 341. Nachbeobachtungstag. Es ergibt sich eine jährliche Schockrate von 2,3\%. Bei 121 Patienten kam es in der Nachbeobachtungszeit zu keinem relevanten Ereignis. Es traten vier inappropriate Schockabgaben auf, die in der statistischen Analyse keine Berücksichtigung fanden.

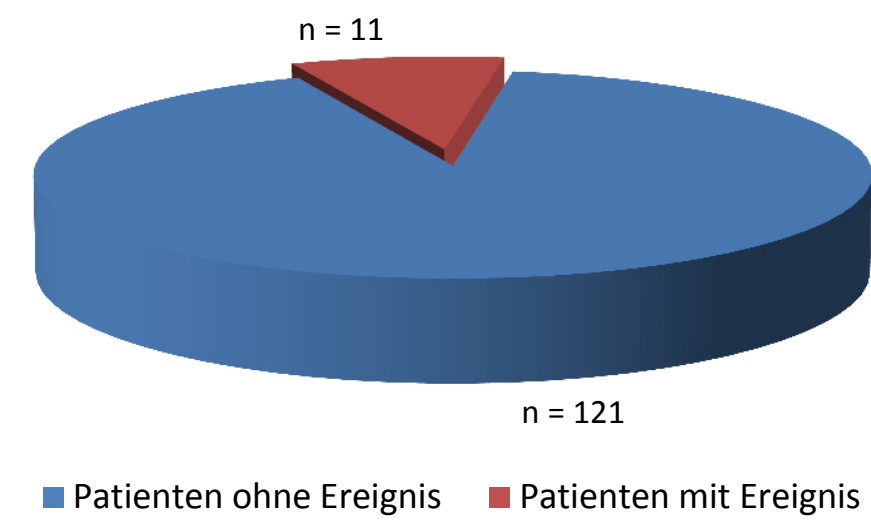

Abb. 8 Anzahl der Patienten mit und ohne Ereignis während des Beobachtungszeitraumes 


\subsection{Erreichen eines Endpunktes in Korrelation mit dem Ergebnis der TWA- Testung}

Bei drei von 63 Patienten mit einer nicht-negativen TWA-Testung trat während der Nachbeobachtungszeit eine appropriate Schockabgabe auf (primärer Endpunkt). Fünf Patienten verstarben (primärer Endpunkt). In der Gruppe mit negativem Testergebnis ( $n=69$ ) wurde kein Schock dokumentiert. Insgesamt verstarben acht Patienten, von denen drei ein negatives Testergebnis aufwiesen. Tabelle 4 zeigte die Ergebnisse der TWA-Testungen in Abhängigkeit von beobachteten primären Endpunkten der Studie. Der Unterschied hinsichtlich appropriater ICD-Schocks zwischen den Patienten mit negativer und denen mit nicht-negativer TWA-Testung konnte als nicht signifikant eingestuft werden (siehe Tabelle 4). Der prädiktive Wert einer negativen TWA-Testung betrug für den Endpunkt „Mortalität" $96 \%$ und für den Endpunkt „appropriate Schockabgabe“ $100 \%$. In einem weiteren Analyseschritt wurden die Endpunkte „appropriate Schockabgabe“ und "Mortalität“ kombiniert betrachtet. Acht Patienten mit nicht-negativem und drei mit negativem Befund erreichten den kombinierten Endpunkt. In der Signifikanzüberprüfung mit einem zweiseitigen exakten Fisher-Test konnte kein signifikanter Unterschied ermittelt werden ( $p=0,12 ;$ siehe Tabelle 4).

\begin{tabular}{|l|c|c|c|}
\hline Endpunkt & $\begin{array}{c}\text { TWA nicht-negativ } \\
\mathbf{n = 6 3 ( 1 0 0 \% )}\end{array}$ & $\begin{array}{c}\text { TWA negativ } \\
\mathbf{n = 6 9 ( 1 0 0} \%)\end{array}$ & $\begin{array}{c}\text { Signifikanz } \\
\text { Fishers exakter Test }\end{array}$ \\
\hline Appropriate Schockabgabe & $3(5 \%)$ & $0(0 \%)$ & $p=0,11$ \\
\hline Mortalität & $5(8 \%)$ & $3(4 \%)$ & $p=0,48$ \\
\hline $\begin{array}{l}\text { Kombinierter Endpunkt } \\
\text { „appropriate Schockabgabe“ } \\
\text { und „Mortalität“ }\end{array}$ & $8(13 \%)$ & $3(4 \%)$ & $p=0,12$ \\
\hline
\end{tabular}

Tab. 4 Erreichen eines Endpunktes in Korrelation mit dem Ergebnis der TWA-Testung 


\subsubsection{Patienten mit appropriater Schockabgabe}

In der Beobachtungszeit trat bei drei Patienten eine appropriate Schockabgabe auf. Alle Patienten, die diesen definierten Endpunkt erreichten, waren aufgrund unbestimmbarer und positiver Testungen (im Verhältnis 2:1) in der Gruppe mit nicht-negativem Testergebnis. Der Grund für die unbestimmbaren Testergebnisse waren jeweils eine unzureichende maximale Herzfrequenz. Die Abbildungen 9 und 10 zeigen Kaplan-MeierÜberlebenskurven für die Zeit bis zum Erreichen des Endpunktes „appropriate Schockabgabe" in Abhängigkeit vom TWA-Befund, wobei zunächst die TWA-Befunde einzeln („positiv“, „negativ“ und „unbestimmbar“) und dann dichotomisiert betrachtet wurden („negativ“ vs. „nicht-negativ“). In der Patientengruppe mit einem unbestimmbaren Testergebnis war das Risiko nach statistischer Auswertung der Überlebenskurven mittels Log-Rank-Test signifikant höher ( $p=0,007)$. Zwischen den Patientengruppen mit positiven und negativen Testergebnissen zeigte sich hinsichtlich der Schockinzidenz kein signifikanter Unterschied (siehe Abbildung 9).

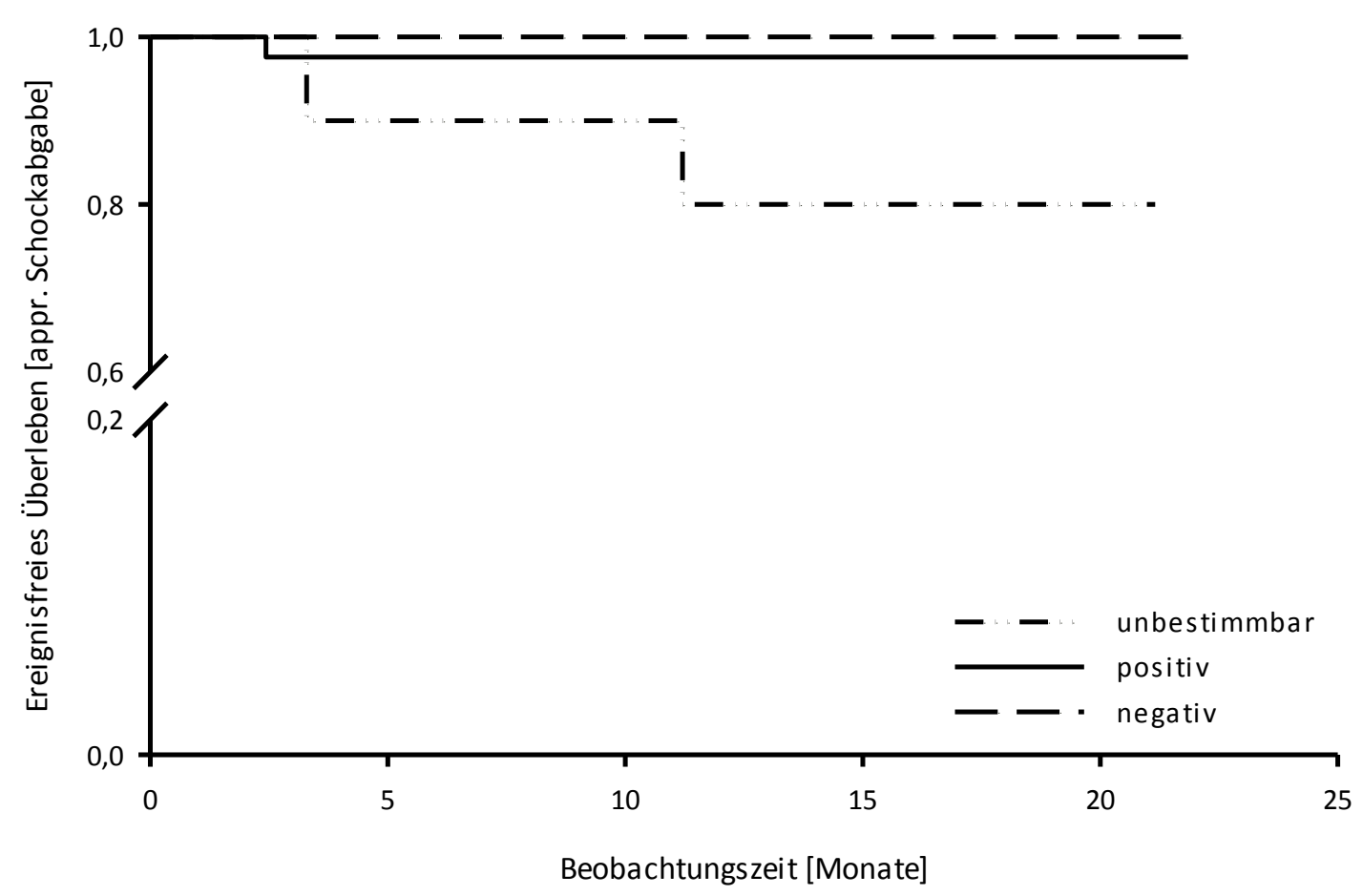

Abb. 9 Ereignisfreies Überleben (hier: „appropriate Schockabgabe“) in Abhängigkeit von einem negativen, positiven bzw. eines unbestimmbaren Testergebnis. Hier zeigte sich kein signifikanter Unterschied zwischen Patienten mit positivem oder negativem Ergebnis. Hingegen hatten Patienten mit einem unbestimmbaren Testergebnis gegenüber Patienten mit negativem Testergebnis ein signifikant höheres Risiko, den Endpunkt „appropriate Schockabgabe“ zu erreichen ( $p=0,007)$. 
In einem zweiten Schritt wurden wie in vielen anderen Studien üblich die Testergebnisse „unbestimmbar“ und „positiv“ zu „nicht-negativ“ zusammengefasst. Mittels Log-Rank-Test konnte für die Wahrscheinlichkeit eines ereignisfreien Überlebens ohne Schock kein signifikanter Unterschied zwischen „nicht-negativ“ und "negativ“ festgestellt werden ( $p=0,08$; siehe Abbildung 10).

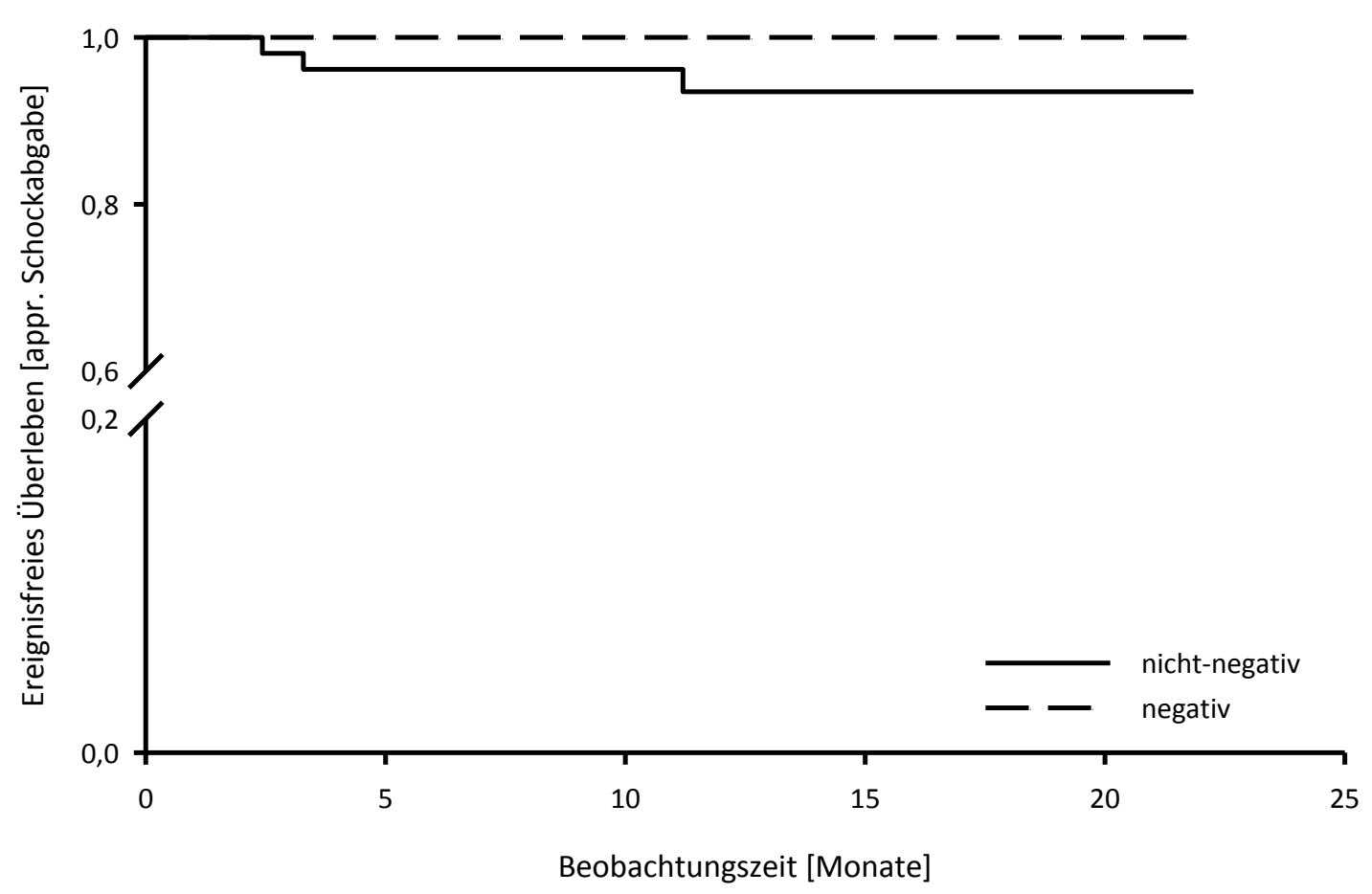

Abb. 10 Ereignisfreies Überleben (hier: „appropriate Schockabgabe“) in Abhängigkeit von einem negativen bzw. eines nicht-negativen Testergebnis $(p=0,08)$.

\subsubsection{Patienten mit dem primären Endpunkt „Mortalität“}

Unter den 132 nachbeobachteten Patienten verstarben im Verlauf acht Patienten. Die Tabelle 5 gibt einen Überblick über diese Patienten und deren TWA-Befund. Von 69 Patienten mit negativem Testergebnis verstarben drei Patienten. Zwei der acht verstorbenen Patienten waren in der 15 Patienten umfassenden Gruppe mit unbestimmbaren TWAErgebnissen. Drei Patienten starben, deren TWA-Testung als „positiv“ gewertet wurde.

\begin{tabular}{|l|c|c|c|c|}
\hline Endpunkt & TWA positiv & $\begin{array}{c}\text { TWA } \\
\text { unbestimmbar }\end{array}$ & $\begin{array}{c}\text { TWA } \\
\text { negativ }\end{array}$ & $\begin{array}{c}\text { TWA nicht-negativ } \\
\text { (,Positive“ + „Unbestimmbare“) }\end{array}$ \\
\hline Gesamtmortalität & $\mathrm{n}=3$ & $\mathrm{n}=2$ & $\mathrm{n}=3$ & $\mathrm{n}=5$ \\
\hline
\end{tabular}

Tab. 5 Verteilung der Testergebnisse der während der Nachbeobachtungszeit verstorbenen Patienten. 
Die Kaplan-Meier-Kurve in Abbildung 11 zeigt die Überlebenswahrscheinlichkeit in Abhängigkeit vom TWA-Befund. In paarweisen Vergleichen konnten keine statistisch signifikanten Unterschiede gefunden werden ( $p_{\text {negativ vs. positiv }}=0,67, p_{\text {unbestimmbar vs. positiv }}=0,18$ und $\left.\mathrm{p}_{\text {unbestimmbar vs. negativ }}=0,23\right)$.

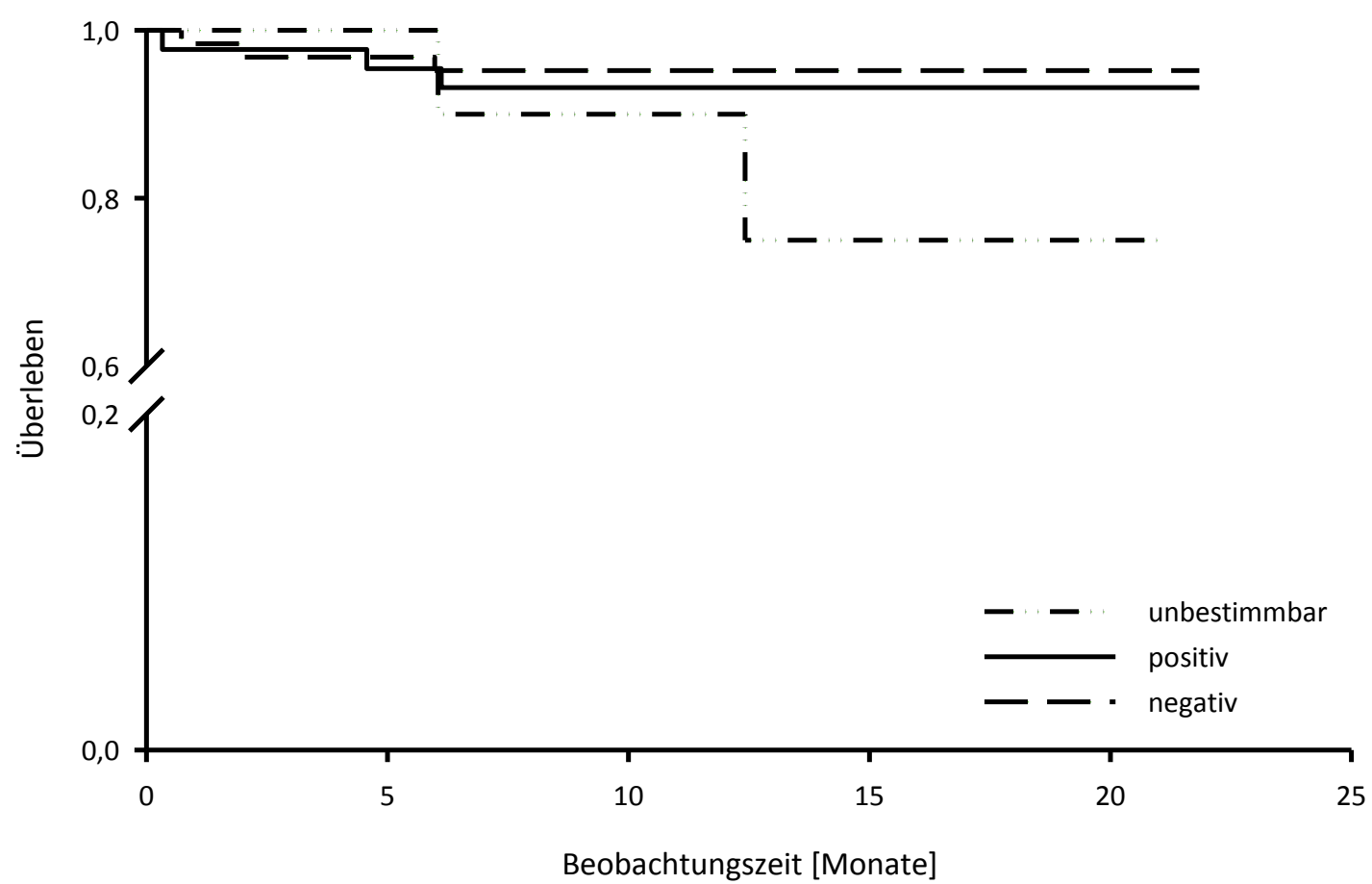

Abb. 11 Überlebenswahrscheinlichkeit in Abhängigkeit vom TWA-Testergebnis („unbestimmbar", "positiv“ bzw. "negativ"). 
Auch für den Endpunkt „Mortalität“ wurden negative TWA-Befunde den nicht-negativen Befunden gegenübergestellt. Im Log-Rank-Test zeigte sich kein signifikanter Zusammenhang zwischen TWA-Befund und "Mortalität" ( $p=0,42$; siehe Abbildung 12).

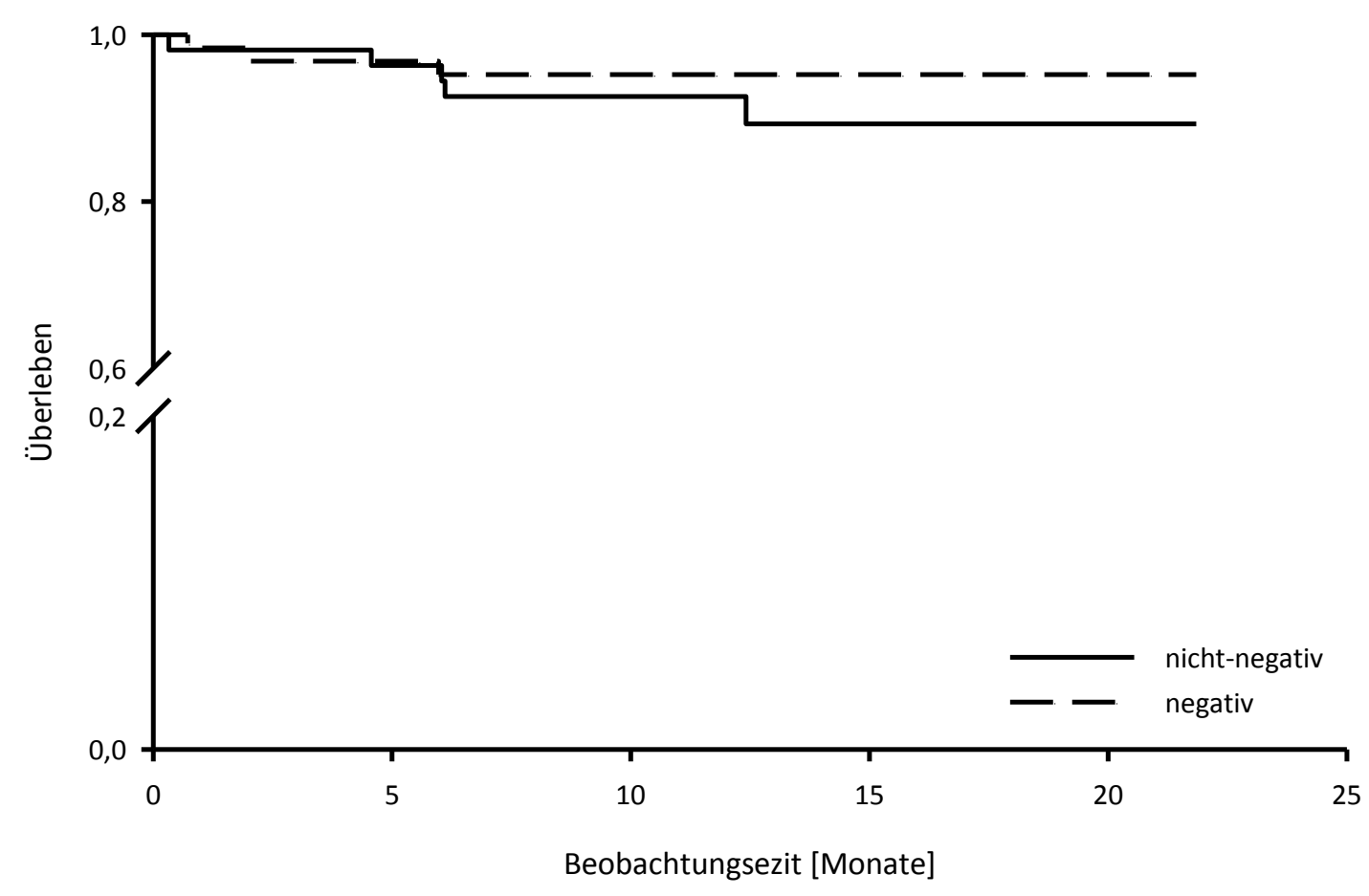

Abb. 12 Überlebenswahrscheinlichkeit in Abhängigkeit von einem negativen bzw. eines nicht-negativen Testergebnis $(p=0,42)$. 


\subsubsection{Kombinierter Endpunkt „appropriate Schockabgabe“ und „Mortalität“}

Abbildung 13 zeigt eine Überlebensanalyse nach Kaplan-Meier, in der das Erreichen des kombinierten Endpunktes „Mortalität“ und „appropriate Schockabgabe“ zwischen den drei Patientengruppen mit negativem, unbestimmbarem und positivem Testergebnis verglichen wurde. In den paarweisen Vergleichen wies der Vergleich zwischen den Gruppen mit unbestimmbarem und negativem Testergebnis eine Signifikanz auf $(p=0,012)$. Die beiden anderen Vergleiche „unbestimmbar“ vs. „positiv“ und „negativ“ vs. „positiv“ waren nicht signifikant unterschiedlich ( $p_{\text {unbestimmbar vs. positiv }}=0,11$ bzw. $p_{\text {negativ vs. posi- }}$ tiv $=0,40)$.

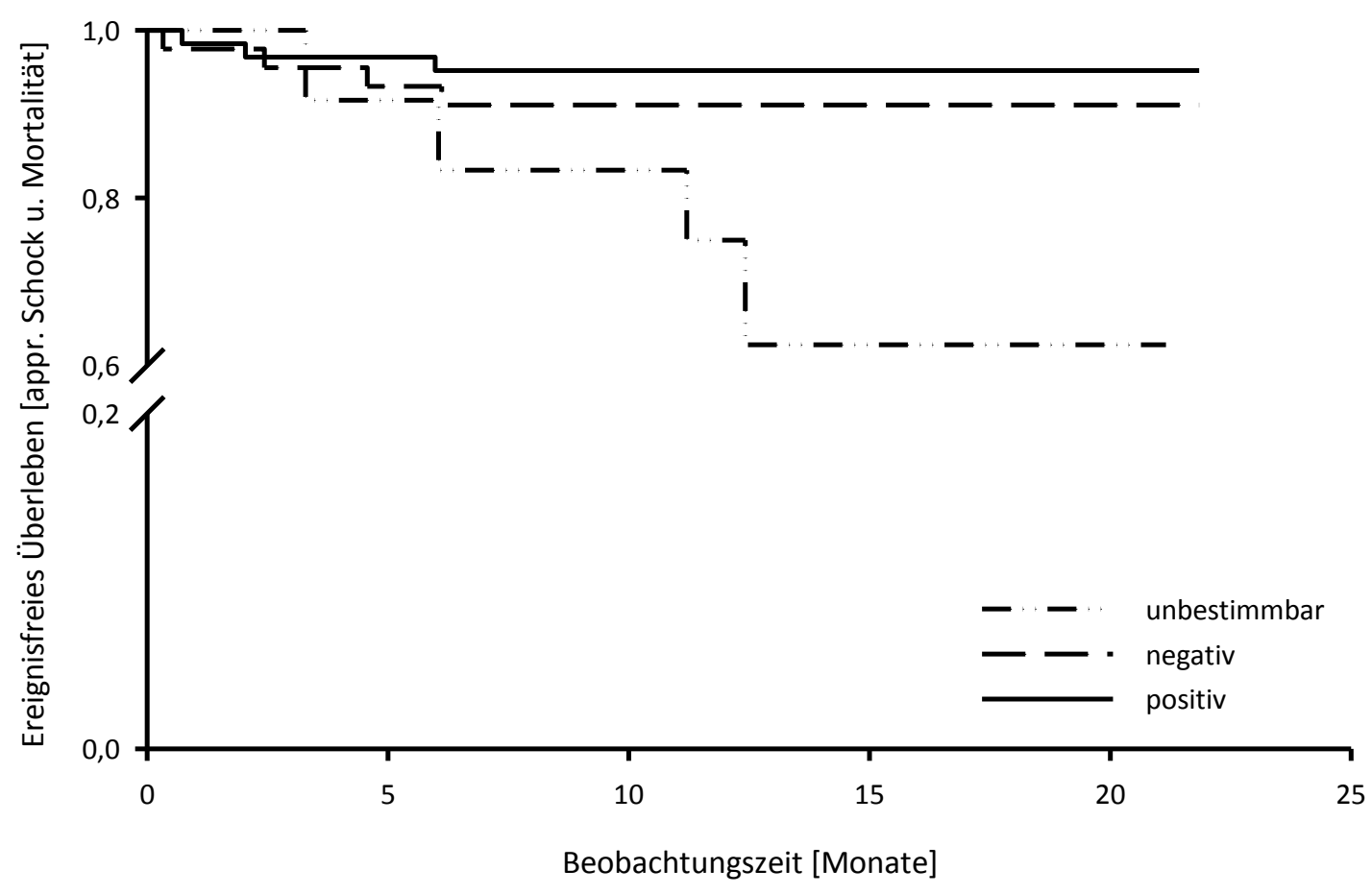

Abb. 13 Ereignisfreies Überleben für den kombinierten Endpunkt „appropriate Schockabgabe“ und "Mortalität“ in Abhängigkeit vom TWA-Testergebnis („unbestimmbar", "negativ“ bzw. "positiv“). Lediglich der Vergleich zwischen unbestimmbaren und negativen Testergbnissen wies eine signifikante Differenz auf $(p=0,012)$. 
Es erfolgte auch eine Analyse des Überlebens im Hinblick auf die zusammengelegten Testergebnisse „unbestimmbar“ und „positiv“ zu „nicht-negativ“ gegenüber den negativen Testergebnissen. Im Gesamtkollektiv zeigte sich kein signifikanter Zusammenhang zwischen TWA-Befund und dem Erreichen des kombinierten Endpunktes ( $p=0,11$; siehe Abbildung 14).

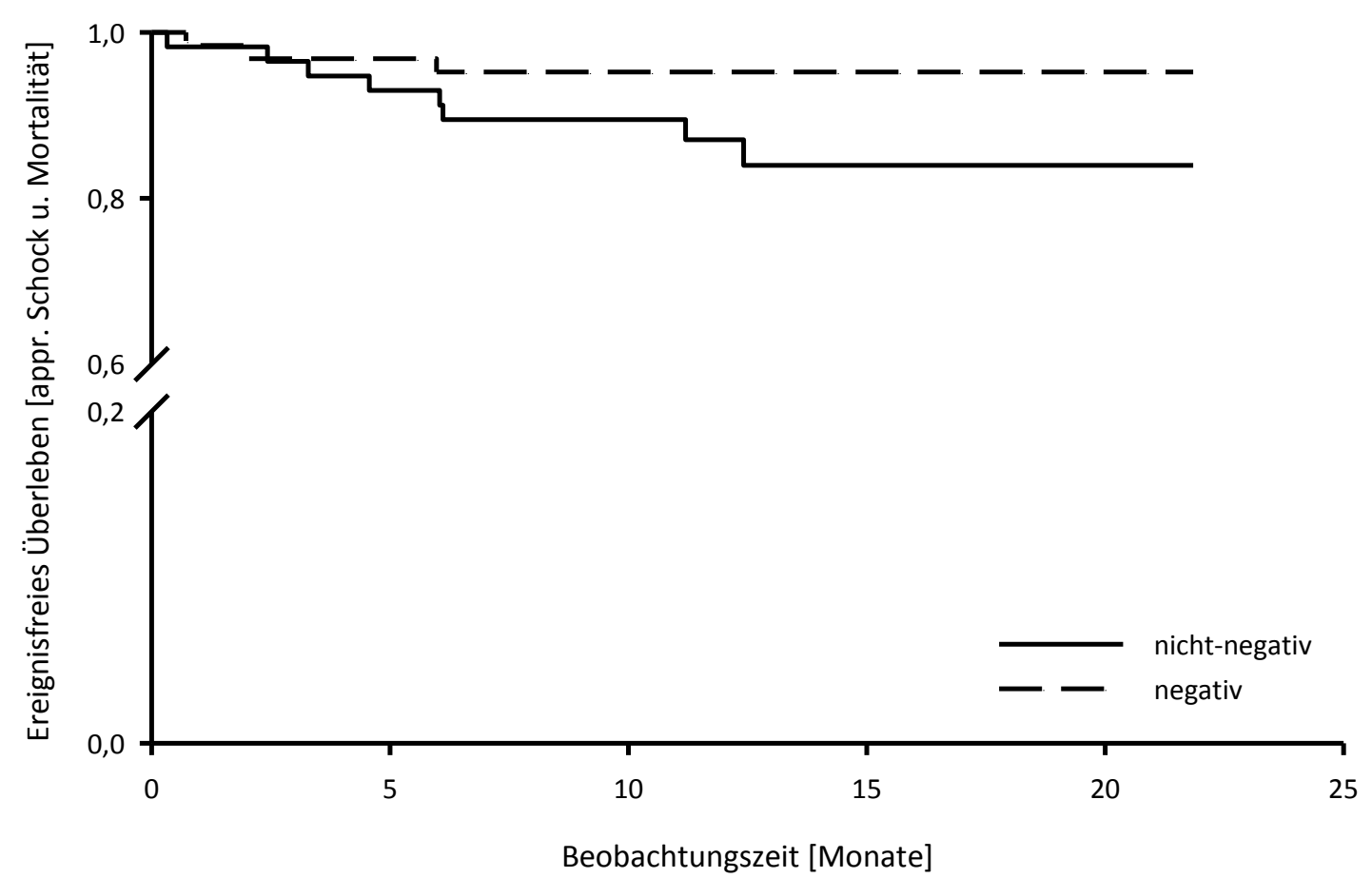

Abb. 14 Überlebenswahrscheinlichkeit und Wahrscheinlichkeit für eine appropriate Schockabgabe in Abhängigkeit vom Vorhandensein eines negativen bzw. eines nicht-negativen Testergebnisses $(p=0,11)$. 


\section{Diskussion}

Ziel der vorliegenden Arbeit war es, im Rahmen einer Beobachtungsstudie, die Bedeutung der TWA-Testung als Risikomarker für lebensbedrohliche ventrikuläre Rhythmusstörungen und für die Gesamtmortalität in einem Patientenkollektiv mit ICD zu ermitteln.

\subsection{Untersuchtes Patientenkollektiv}

In den bisherigen klinischen Arbeiten zur Bedeutung des MTWA in der Risikostratifikation für den SCD erfolgte eine Selektion der Patienten nach der kardialen Grunderkrankung und/oder nach dem Grad der LVEF-Einschränkung.

Chow et al. (2007) beispielsweise schlossen 768 Patienten mit einer ischämischen Kardiomyopathie (ICM) und einer LVEF $\leq 35 \%$ in ihre prospektive Studie ein. Kein Patient hatte vor Einschluss ein Ereignis mit einer anhaltenden ventrikulären Arrhythmie. 392 (51 \%) Patienten wurde ein ICD implantiert. In diesem Patientenkollektiv erwies sich der MTWA als starker und unabhängiger Prädiktor für die Gesamtmortalität und letale Arrhythmien. Zur Verhinderung eines Todesfalles innerhalb von zwei Jahren mussten neun Patienten mit nicht-negativem Testergebnis mit einem ICD versorgt werden (NNT), wohingegen die NNT unter Patienten mit negativem Testergebnis 76 betrug. In der 446 Patienten umfassenden prospektiven Multizenterstudie ALPHA (T-wave alternans in patients with heart failure) waren auffällige TWA-Testungen bei Patienten mit nichtischämischer DCM (NYHA II/III, LVEF $\leq 40 \%$ ) mit einem vierfach höheren Risiko für einen kardial bedingten Tod und lebensbedrohliche Arrhythmien assoziiert (HR 4,00, 95 \% Konfidenzintervall, 1,4-11,4 \%, $p=0,002$ ). Von den 446 Patienten wiesen 154 (35\%) Patienten einen negativen TWA-Befund und 446 (65\%) einen nicht-negativen Befund auf. Anders als bei der eben erwähnten Studie von Chow et al. (2007) stellte eine stattgehabte Implantation eines ICD ein Ausschlusskriterium dar. Die Studie wurde ausschließlich zur Untersuchung der primärpräventiven Wertigkeit der TWA-Testung durchgeführt. Folglich war kein Patient bei Einschluss mit einem ICD versorgt (Salerno-Uriarte et al. 2007).

Die Arbeitsgruppe um Bloomfield veröffentlichte 2006 eine Arbeit, in der 549 Patienten mit einer ischämischen oder einer nichtischämischen Kardiomyopathie und einer LVEF $\leq 40 \%$ eingeschlossen wurden. 69 (13\%) Patienten waren mit einem ICD versorgt (acht vor und 61 Patienten nach Einschluss). Wie in der vorliegenden Arbeit wurden "Gesamtmortalität“ und „appropriate ICD-Schockabgabe“ als Endpunkte gewertet. Insgesamt erreichten während der zweijährigen Nachbeobachtungszeit 47 (13\%) der 360 Patienten mit nicht-negativem TWA-Befund einen Endpunkt, was einer Ereignisrate von 15 entspricht. Einzeln betrachtet war die Ereignisrate bei den Patienten mit positivem Testergebnis ( $n=162$ ) mit $12,3 \%$ niedriger als bei den Patienten mit unbestimmbarem Testergebnis $(n=198)$. Hier betrug sie $17,5 \%$. Dahingegen erreichten lediglich vier (2 \%) Patienten der 189 Patienten mit unauffälligem Testergebnis einen Endpunkt. Die Ereignisrate beträgt 2,5. Um welchen der beiden Endpunkte es sich handelte, ist aus der Arbeit nicht ersichtlich. 
In der vorliegenden Arbeit wurden prospektiv, monozentrisch und konsekutiv Patienten mit ICD-Erstimplantation untersucht. Die Patientenselektion wurde weder durch die kardiale Grunderkrankung noch durch die Indikation für eine ICD-Implantation beeinflusst. Patienten mit einer ICM oder mit einer DCM stellten den größten Anteil des untersuchten Kollektivs (54\% ICM und $25 \%$ DCM) dar. Dazu kamen $2 \%$ mit einer arrhythmogenen rechtsventrikulären Kardiomyopathie, $2 \%$ mit hypertropher Kardiomyopathie und $1 \%$ mit einer hypertensiven Herzerkrankung. In die Studie von Chow et al. wurden ausschließlich Patienten mit einer ICM und in die ALPHA-Studie nur Patienten mit nichtischämischer Kardiomyopathie eingeschlossen. In der Arbeit von Bloomfield et al. wurde sowohl Patienten mit ischämischer (49\%) als auch nichtischämischer Kardiomyopathie berücksichtigt (Bloomfield et al. 2006; Chow et al. 2007; Salerno-Uriarte et al. 2007).

88 (66\%) der in dieser Arbeit untersuchten Patienten haben eine lebensbedrohliche Rhythmusstörung überlebt. Die übrigen Patienten hatten keine Rhythmusstörung dieser Art vor der Implantation und somit vor der TWA-Testung. Knapp $22 \%$ der eingeschlossenen Patienten wiesen eine LVEF von $\geq 40 \%$ auf. Bei 104 von 134 Patienten (78\%) betrug sie $\leq 35 \%$. Diese Patienten stellten somit einen erheblichen Anteil am Gesamtkollektiv dar.

Zusammenfassend lässt sich das hier untersuchte Patientenkollektiv somit als ein gemischtes Kollektiv mit leitlinientypischen primär- und sekundärpräventiven ICD-Indikationen beschreiben. Es spiegelt daher den klinischen Alltag von Patienten mit hohem Risiko für maligne Rhythmusstörungen und Indikation zur ICD-Implantation wider. In dieser eher heterogenen Patientengruppe wäre klinisch eine Differenzierung von ICD-Schock- und Mortalitätsrisiko wertvoll.

\subsection{Verteilung der Testergebnisse im nichtselektierten Patientenkollektiv}

Es erfolgte die Auswertung von insgesamt 134 TWA-Testungen. Die Testungen wurden gemäß den 2002 standardisierten Interpretationsrichtlinien bewertet (Bloomfield et al. 2002) und so in positive, negative und unbestimmbare Ergebnisse eingeteilt: Es fanden sich 50 positive (37\%), 69 negative (52\%) und 15 unbestimmbare (11\%) TWA-Testungen. In früheren Arbeiten mit TWA-Testungen wurden sowohl ähnliche als auch abweichende Verteilungen beobachtet. Ikeda et al. (2006) untersuchten den prädiktiven Wert des MTWA bei 1.003 Patienten nach einem Myokardinfarkt und einer LVEF $\geq 40 \%$. Hier betrug der Anteil der positiven Testungen $17 \%$, derjenige der negativen Testungen $74 \%$ und der Anteil der unbestimmbaren Testungen $9 \%$. Aufgrund einer höheren Ereignisrate an arrhythmischen Ereignissen bei positiv getesteten Patienten hielten Ikeda et al. (2006) den MTWA für einen sinnvollen Risikomarker. Adachi et al. (2001) untersuchten 82 Patienten mit einer nichtischämischen DCM mit TWA-Testungen. Die Verteilung der Ergebnisse war wie folgt: Die TWA-Testung war bei 30 Patienten positiv (37\%), bei 34 negativ (41\%) und bei 18 unbestimmbar (22\%).

Die TWA-Testungen der oben erwähnten Arbeiten erfolgten auch mit dem $\mathrm{CH}_{2000}{ }^{\mathrm{TM}}$ und die Interpretation nach den gleichen Kriterien. Ein direkter Vergleich ist jedoch nicht 
möglich. Es ist bekannt, dass herzwirksame Medikamente wie Beta-AdrenorezeptorAntagonisten (sogenannte Beta-Blocker) die Alternansspannung vermindernd beeinflussen (Klingenheben et al. 2001; Rashba et al. 2002). Folglich kann eine TWA-Testung mit Beta-Blockern „negativ“ und eine Testung ohne Beta-Blockereinnahme „positiv“ ausfallen (Klingenheben et al. 2001). In einigen Arbeiten wurde vor der TWA-Testung die Einnahme von Beta-Blockern pausiert (Baravelli et al. 2005; Gold et al. 2000), um die zur Testung erforderliche Herzfrequenzanhebung leichter zu erreichen. Knapp $90 \%$ der Patienten des hier untersuchten Kollektivs nahmen täglich Beta-Blocker ein. Vor den routinemäßig durchgeführten TWA-Testungen dieser Arbeit wurde auf eine Veränderung der Medikation verzichtet, da ein plötzliches Absetzen zu gefährlichen Rhythmusstörungen führen kann (Rebound-Effekt) und die protektive Wirkung unterbrochen wird (Eber et al. 2000). Wird die frequenzmodulierende Therapie vor TWA-Testung unterbrochen, so würde der Risikomarker MTWA zu einem Zeitpunkt bestimmt werden, an dem der Patient per se einem erhöhten Mortalitätsrisiko ausgesetzt ist. Zudem sollte eine Risikostratifikation bezogen auf eine tatsächlich im klinischen Alltag vorliegende Situation vorgenommen werden. Auch andere einschlägige Studien zur Bedeutung der TWA-Testung sahen von einer Medikationsveränderung zur TWA-Testung ab (Bloomfield et al. 2006; Chow et al. 2007; Ikeda et al. 2006; Salerno-Uriarte et al. 2007; Tapanainen et al. 2001).

Klingenheben et al. (2001) und Myles et al. (2007) empfahlen, eine TWA-Testung unter Beibehaltung der herzwirksamen Medikation vorzunehmen und begründeten dies ebenfalls mit der Tatsache, nur so ein tatsächliches aktuelles Risiko für den SCD ermitteln zu können. Chan et al. (2007) zeigten in einer Metaanalyse mit neun Studien, dass eine TWA-Testung eine höhere Sensitivität und einen besseren negativen prädiktiven Wert aufweist (npW), wenn die Therapie mit Beta-Blockern nicht unterbrochen wird.

Ferner können nichtkardiale Begleiterkrankungen wie beispielsweise eine Nierenfunktionseinschränkung das Ergebnis der TWA-Testung beeinflussen. Secemsky et al. (2011) berichteten in ihrer Veröffentlichung von einer erhöhten Prävalenz von TWA in einem 72-stündigen Holter-EKG bei Dialysepatienten. Obwohl Patienten mit Diabetes mellitus ein erhöhtes Risiko für den SCD haben, insbesondere wenn ein Myokardinfarkt überlebt wurde, war die Prävalenz auffälliger TWA-Testungen bei dieser Patientengruppe nicht höher (Martin et al. 2009). Diese Beispiele verdeutlichen, dass es wichtig ist, den Einfluss nichtkardialer Begleiterkrankungen zukünftig zu untersuchen, um schließlich einen direkten Vergleich der verschiedenen Arbeiten über den MTWA als Prädiktor zu ermöglichen.

\subsection{Ergebnisüberblick}

Mit Hilfe der vorliegenden Studie zur Risikoprädiktion durch TWA konnte gezeigt werden, dass Patienten nach ICD-Erstimplantation und unbestimmbarer TWA-Testung eher eine appropriate Schockabgabe erhielten als Patienten mit einer negativen und somit unauffälligen Testung. Wie in anderen Studien zur Bedeutung der TWA-Testung üblich, wurden positive und unbestimmbare Testungen zu „nicht-negativ“ zusammengefasst und der 
Gruppe mit negativen Testungen gegenübergestellt. Hier zeigte sich jedoch kein signifikanter Unterschied hinsichtlich der Schockinzidenz. In der polnischen Studie von Lewandowski et al. (2011) mit vergleichbarem Studiendesign konnte nach einer Nachbeobachtungszeit von $12 \pm 6$ Monaten bei 67 Patienten mit ICD (31 primärpräventiv und 36 sekundärpräventiv implantiert) nach TWA-Testung ebenfalls kein statistisch signifikanter Unterschied bezüglich der Schockinzidenz bei dem Vergleich der Gruppen mit nichtnegativem und negativem Befund gefunden werden. Bei zehn der 35 Patienten mit nichtnegativem Testergebnis konnte eine ventrikuläre Tachyarrhythmie registriert werden ( $p=$ nicht signifikant). Auch nicht signifikant war der Unterschied bei den Patienten mit negativer Testung. Sechs ICDs der 32 Patienten mit negativer TWA-Testung zeichneten eine tachyarrhythmische ventrikuläre Phase auf. Wie viele ventrikuläre Tachyarrhythmien mittels Schock und wie viele mit ATP therapiert wurden, ist aus der Arbeit nicht ersichtlich. Anders als in dieser Arbeit wurde die Einnahme von Beta-Blockern 12 bis 24 Stunden vor TWA-Testung pausiert. Während das Durchschnittsalter des Patientenkollektivs der Studie von Lewandowski et al. (2011) etwa dem hier untersuchten Kollektiv entsprach, war die gemittelte LVEF des in dieser Arbeit untersuchten Kollektivs rund $10 \%$ niedriger gegenüber der Patientengruppe mit nicht-negativer Testung (34 $\pm 9 \%$ ) und circa $20 \%$ niedriger gegenüber der Gruppe mit negativer Testung (38 $\pm 11 \%$ ). Bei vergleichbarer Nachbeobachtungszeit kam es in der polnischen Studie zu 16 ICD-Therapien (Schock, ATP und intrakardiale Konversion) und in dieser Arbeit zu drei appropriaten ICD-Schocks. Hierbei ist in jedem Fall zu berücksichtigen, dass ATPs und intrakardiale Konversionen keine statistische Berücksichtigung fanden. Neben demographischen Unterschieden und etwas ungleichen Endpunktdefinitionen muss bei der Beurteilung der Anzahl der erreichten Endpunkte die Programmierung des ICD beim Vergleich von Studien zur Bedeutung der TWA-Testung einbezogen werden. Die VT-Detektionsrate begann bereits bei 200 Schlägen/min, wohingegen am Herzzentrum Göttingen in der klinischen Routine die VF-Zone bei 220 bis 230 Schlägen/min programmiert wird.

Die durchschnittliche Anzahl ereigneter Schocks war mit 2,3\% pro Jahr geringer als in einigen anderen Studien mit ähnlicher Fragestellung, obwohl etwa jede dritte ICDImplantation einer sekundärpräventiven Maßnahme entsprach. Wilson et. al (2012) konnten zeigen, dass es bei sekundärpräventiv implantierten ICDs eher zu einem appropriaten Schock kommt als bei einer primärpräventiven Implantation (HR 2,11, 95 \% Konfidenzintervall, 1,16-3,85, $p=0,01$ ). Nach sieben Jahren Nachbeobachtung wurde bei $55 \%$ der Patienten eine appropriate ICD-Therapie registriert (7,9\% pro Jahr). In der SCD-HeFtStudie wurde eine durchschnittliche jährliche Rate appropriater Schocks von 5,1\% pro Jahr bei einer medianen Beobachtungszeit von 45,5 Monaten verzeichnet (Bardy et al. 2005). Obwohl in der SCD-HeFt-Studie die Implantation ausschließlich zur Primärprävention durchgeführt wurde, war die durchschnittliche jährliche Rate an appropriaten Schocks etwa doppelt so hoch wie im Patientenkollektiv dieser Arbeit. Die Ursache hierfür ist sicherlich unter anderem im Patientenkollektiv zu suchen. Das Risiko für das Auftreten 
lebensbedrohlicher tachykarder Herzrhythmusstörungen bei Patienten mit einer ventrikulären Funktionsstörung wird durch eine medikamentöse Therapie und durch die Patientenbetreuung beeinflusst. Die geringe Anzahl an Schocks bei dem hier untersuchten Patientenkollektiv lässt die mögliche Schlussfolgerung zu, dass die Patienten optimal medikamentös und nicht-medikamentös (beispielsweise durch Compliance-fördernde Maßnahmen) therapiert wurden. Während in dem Göttinger Kollektiv ein ATP möglich war, um die Abgabe unnötiger und oft folgenschwerer Schockabgaben zu vermeiden, war diese ICD-Funktion beispielsweise im SCD-HeFT-Kollektiv ausgeschaltet. Folglich konnten einige ventrikuläre Tachykardien, die im Göttinger Kollektiv durch ein ATP therapiert wurden, gar nicht oder nur mittels ICD-Schock therapiert werden, was somit zur Erhöhung der Schockinzidenz führt. Alle ICDs wurden mit einer durchaus geringen Detektionsrate von $\geq 187$ Schlägen/min programmiert, was 43 Schläge/Minute unterhalb der unteren Grenze der üblichen Detektionsrate des hier untersuchten Kollektivs entspricht.

Auch bei der Bewertung der Gesamtmortalität ist neben der Anzahl der ICD-Schocks auch die ATP-Funktion zu beachten. ICD-Schocks gehen mit einer erhöhten Mortalität einher und können so die Anzahl der statistisch berücksichtigen Todesfälle beeinflussen, sofern der erste Schock nicht zum Ausscheiden aus dem Patientenkollektiv führt, wie in dieser Arbeit (Borne et al. 2013; Larsen et al. 2011). Ähnliches gilt für das ATP. Die recht neue 1.500 Patienten umfassende MADIT-RIT-Studie zeigte, dass auch das Auftreten von ATP die

Mortalitätsrate erhöhen kann. Nach einer durchschnittlichen Nachbeobachtungszeit von 1,4 Jahren war die Gesamtmortalitätsrate der Patientengruppe mit konventionell programmiertem ICD (mit ATP-Funktion) höher als in der Gruppe, in der die ICDs Detektionsrate $\geq 200$ Schlägen/Minute und einer 2,5-sekündigen Verzögerung bis zum Auslösen eines Schockes programmiert waren (HR 0,45, 95\% Konfidenzintervall, 0,24-0,85, $p=0,01$ ) (Moss et al. 2012).

Die Gesamtmortalität in der vorliegenden Arbeit lag in der Patientengruppe mit nichtnegativer Testung höher, jedoch ohne statistisch signifikanten Unterschied gegenüber den Patienten mit einer negativen Testung. Es ließ sich zeigen, dass Patienten mit einer unbestimmbaren Testung ein signifikant höheres Mortalitätsrisiko trugen als Patienten mit einer negativen Testung. In der Gruppe mit positiven Testungen traten nicht signifikant mehr Todesfälle auf als in der Gruppe mit unbestimmbaren oder negativen Testungen. In der von Bloomfield et al. (2004) durchgeführten Studie zur Bedeutung der TWA-Testung, in der die Patienten die MADIT-II-Kriterien erfüllten, verstarben jährlich durchschnittlich 8,9 \% Patienten mit einer auffälligen TWA-Testung (positiv oder unbestimmbar) und 1,9\% mit einer negativen Testung. In dem in dieser Arbeit untersuchten Patientenkollektiv starben 8 \% bzw. 4 \% jährlich. Obwohl die LVEF der Patienten des Bloomfield-Kollektivs mit durchschnittlich $23 \pm 6 \%$ rund $10 \%$ unter der mittleren Ejektionsfraktion dieses Kollektivs lag, war die Mortalität vergleichbar hoch. In der Zusammenschau scheint eine unbestimmbare Testung ein stärkerer Prädiktor für die Gesamtmortalität zu sein als bisher 
angenommen (siehe Kapitel 4.4.). Salerno-Uriarte et al. (2007) untersuchten 464 Patienten mit einer nicht-ischämischen Kardiomyopathie und einer LVEF $\leq 40 \%$ mittels TWATestung. Den sekundären Endpunkt „Gesamtmortalität" erreichten durchschnittlich jährlich ungefähr $5 \%$ der Patienten mit einer nicht-negativen TWA-Testung und nur etwa $1 \%$ mit unauffälliger Testung. Während die LVEF in diesem Kollektiv etwa der LVEF des in dieser Arbeit untersuchten Kollektivs entsprach, war hingegen das Alter der Patienten, gemittelt betrachtet, circa fünf Jahre niedriger.

Im Vergleich zu den oben aufgeführten Studien ist die Häufigkeit der Gesamtmortalität als Endpunkt nicht wesentlich unterschiedlich, jedoch die Häufigkeit appropriater Schockabgaben niedriger. Die Aussagekraft dieser Untersuchung ist jedoch bei einer geringen Fallzahl und relativ kurzer Nachbeobachtungszeit limitiert und lässt daher eine Etablierung der TWA-Testung als effiziente Risikostratifikationsmethode mit sicherer prognostischer Wertigkeit für maligne Herzrhythmusstörungen und Mortalität im deutschen, klinischen Alltag nicht zu. Aufgrund der geringen Fallzahl dürfen die Resultate bloß als mögliche Tendenz gewertet werden.

\subsection{Bedeutung von unbestimmbaren TWA-Testungen}

In der vorliegenden Arbeit wurden für die Auswertung der TWA-Testungen wie allgemein üblich die von Bloomfield et al. (2002) publizierten Interpretationsrichtlinien verwendet. Demnach ist eine Klassifikation in „positiv“, „negativ“ und „unbestimmbar" möglich. Eine TWA-Testung wird als „unbestimmbar" bezeichnet, sofern weder die Kriterien für eine positive noch für eine negative TWA-Testung erfüllt werden. Während die Bedeutung für ein positives oder ein negatives Ergebnis leicht nachvollzogen werden kann, ist sie für unbestimmbare Testergebnisse zunächst nicht offensichtlich.

Kaufmann et al. (2006) untersuchten die Bedeutung von unbestimmbaren TWATestungen. Unter Berücksichtigung von sechs Arbeiten ermittelten sie, dass 20 bis $40 \%$ der TWA-Testungen unbestimmbar sind. In der ABCD-Studie mit 566 Patienten lag der Anteil an unbestimmbaren Testungen bei $25 \%$ (Costantini et al. 2009). Die Entstehung unbestimmbarer Testergebnisse ist dabei auf zwei Hauptfaktoren zurückzuführen. Zum einen sind es patientenabhängige Faktoren, wie z. B. eine zu geringe maximal erreichte Herzfrequenz, ein hoher Anteil an Extrasystolen oder beeinflussende Muskel- und Bewegungsartefakte. Zum anderen sind auch technische Faktoren limitierend. Hier sind als Beispiel Elektrodenlockerungen und Fehlfunktionen der Kabel zu erwähnen. Die patientenabhängigen Faktoren können im Gegensatz zu technischen Faktoren einen eigenen prädiktiven Wert enthalten, worauf man im Allgemeinen die schlechte Prognose von Patienten mit unbestimmbarer TWA-Testung zurückführt.

In der vorliegenden Arbeit waren 15 von 134 TWA-Testungen (11\%) unbestimmbar. Somit lag die Rate an unbestimmbaren Testungen unter der durchschnittlichen Rate. Ein Grund hierfür könnte sein, dass bei jeder Testung besonders große Sorgfalt auf die Vermeidung von Muskel- und Bewegungsartefakten gelegt wurde. Des Weiteren wurde eine gründliche 
Hautpräparation vorgenommen. Die TWA-Testungen wurden erst gestartet, nachdem alle Elektroden eine optimale Konnektivität aufwiesen (siehe Kapitel 2.3.1).

Wie oben erwähnt, untersuchten Kaufman et al. (2006) die Bedeutung der unbestimmbaren TWA-Testungen bei Patienten mit einer LVEF $\leq 40 \%$. Sie konnten zeigen, dass aufgrund von ventrikulären Extrasystolen oder einer zu geringen maximalen Herzfrequenz unbestimmbare Testungen genauso gut die Gesamtmortalität und anhaltende ventrikuläre Arrhythmien vorhersagen können wie positive TWA-Testungen. Die Ergebnisse der Arbeit von Kaufman et al. (2006) erlauben eine Zusammenfassung positiver und unbestimmbarer TWA-Testungen in eine Gruppe mit nicht-negativen Testungen, da unbestimmbare TWA-Testungen bei Patienten mit einer ventrikulären Funktionseinschränkung genau so gut Mortalität und anhaltende ventrikuläre Arrhythmien vorhersagen wie positive TWA-Testungen, sofern die Ursachen für die unbestimmbare TWA-Testung patientenabhängig sind (unzureichende maximale Herzfrequenz, zu hoher Anteil an ventrikulären Extrasystolen) und nicht technisch bedingt sind (Signal/Rauschverhältnis zu Ungunsten des Signals). Chan et al. (2007) kamen nach ihren Untersuchungen zu dem Entschluss, dass eine Klassifizierung in krankhafte („nicht-negative“) und unauffällige („negative“) TWA-Testungen den prädiktiven Wert verbessert, da unbestimmbare TWA-Testungen aufgrund eines zu hohen Anteils an ektopen Herzschlägen oder einer unzureichenden Herzfrequenz mit einem signifikanten Risiko für Gesamtmortalität und letalen Herzrhythmusstörungen einhergehen (HR 4,63, 95\% Konfidenzintervall, 1,32-16,18, $p=0,02$ ).

In dem von uns untersuchten Patientenkollektiv trat bei drei Patienten eine appropriate Schockabgabe während der Nachbeobachtung auf, wovon zwei Drittel aufgrund einer zu geringen maximal erreichten Herzfrequenz eine unbestimmbare TWA-Testung aufwiesen. Jeder vierte verstorbene Patient während der Nachbeobachtungszeit hatte eine unbestimmbare TWA-Testung.

Nach dem Vorbild der ABCD-Studie und einiger anderer Veröffentlichungen wurde auch in der vorliegenden Arbeit zwischen negativen und nicht-negativen TWA-Testungen differenziert (Chow et al. 2007; Costantini et al. 2009). Hier zeigte sich dann jedoch kein signifikanter Unterschied, wohingegen in der Einzelbetrachtung Patienten mit unbestimmbaren Testergebnis ein signifikant höheres Risiko hatten, den Endpunkt „appropriater Schock“ zu erreichen als Patienten mit negativem Befund ( $p=0,007)$. In dieser Arbeit waren hauptsächlich eine unzureichende maximale Herzfrequenz (53\%) und ein zu hoher Anteil ektoper Herzschläge (27\%) Ursachen für unbestimmbare TWA-Testungen. In der Zusammenschau mit den gewonnen Daten der vorliegenden Arbeit lässt sich vermuten, dass die Bedeutung der unbestimmbaren Testungen bisher unterbewertet ist und eine Analyse der unbestimmbaren TWA-Testungen sinnvoll erscheint, insbesondere wenn eine patientenabhängige Ursache zum entsprechenden Testergebnis führt. In der Studie von Daniłowicz-Szymanowicz et al. (2012) mit 93 Patienten (63 \pm 13 Jahre, LVEF $30 \pm 7 \%$ ) betrug die Anzahl unbestimmbarer Testungen 25 (27\%). Hiervon waren 15 TWA- 
Testungen aufgrund einer patientenabhängigen Ursache und fünf aus technischen Gründen als „unbestimmbar“ gewertet worden. Während der $10 \pm 6$-monatigen Nachbeobachtungszeit wurde achtmal einer der Endpunkte SCD, VT oder VF erreicht. In der Gruppe mit nicht-negativem TWA-Befund erreichten signifikant mehr Patienten einen Endpunkt als Patienten mit negativem TWA-Befund ( $p=0,007)$. Innerhalb der Gruppe mit nichtnegativem Befund war die Wahrscheinlichkeit einen der genannten Endpunkte (SCD, VT, VF) zu erreichen ähnlich, sofern die unbestimmbaren TWA-Testungen patientenabhängiger Ursache waren. Waren die unbestimmbaren TWA-Testungen technisch bedingt oder verblieb die TWA-Testung negativ, so war das Risiko für Patienten mit positiver TWATestung signifikant höher $(p<0,05)$. Anscheinend haben die technisch bedingten unbestimmbaren Testungen einen wesentlich geringeren prognostischen Wert und unbestimmbare TWA-Testungen aufgrund einer patientenabhängigen Ursache einen sehr hohen Wert, der mit einer positiven TWA-Testung vergleichbar ist. Die Daten der vorliegenden Arbeit unterstützen dies.

Ikeda et al. (2006) ermittelten einen npW von 99,6 \% für die TWA-Testung bei Patienten nach Myokardinfarkt und einer LVEF $\geq 40 \%$ (vgl. Kapitel 4.2). In der statistischen Analyse konnte die TWA-Testung als hochsensitive Risikoprädiktion für lebensbedrohliche arrhythmische Ereignisse gewertet werden (HR 19,7, 95 \% Konfidenzintervall, 5,5-70,4; $p<0,0001$ ). Myles et al. (2007) gaben in einer ausführlichen Übersichtsarbeit mit 17 Studien unterschiedlicher Patientenkollektive einen durchschnittlichen npW zwischen $90 \%$ und $100 \%$ an. Eine Ausnahme bildete die Arbeit von Rashba et al. (2004) mit 144 KHK-Patienten (LVEF $\leq 40 \%$ ). Hier betrug der npW lediglich $84 \%$. Aufgrund des hohen $\mathrm{npW}$ in verschiedenen Kollektiven ist es plausibel, die negativen mit den nicht-negativen TWA-Testungen zu vergleichen. Die Daten der vorliegenden Arbeit verhalten sich hinsichtlich des npW für anhaltende ventrikuläre Arrhythmien wie bei Myles et al. (2007). Kein Patient mit einer negativen TWA-Testung hatte eine ventrikuläre Arrhythmie, die mit einem appropriaten Schock therapiert werden musste ( $n p W=100 \%)$. Dahingegen konnte keine Signifikanz für eine positive TWA-Testung gefunden werden, wie es zum Beispiel bei Ikeda et al. (2006) der Fall war $(p<0,0001)$. Laut MASTER-Studie (Microvolt T Wave Alternans Testing for Risk Stratification of Post-Myocardial Infarction Patients) mit 575 Myokardinfarktpatienten (LVEF $\leq 30 \%$ ) (214 Patienten (37\%) mit positiver, 293 Patienten (51\%) mit negativer und 68 Patienten (12\%) mit einem unbestimmbaren TWA-Ergebnis) ist die TWA-Testung keine geeignete Methode, um für ventrikuläre Tachyarrhythmien zu stratifizieren. Ein signifikanter Unterschied zwischen Patienten mit negativer und nichtnegativer TWA-Testung zeigte sich nicht (HR 1,26, 95\% Konfidenzintervall, 0,76-2,09; $p=0,37)$. Anders verhielt es sich hinsichtlich des Endpunktes „Mortalität“. Hier ließ sich ein signifikanter Unterschied finden. Patienten mit einer auffälligen TWA-Testung hatten ein größeres Risiko in der zweijährigen Nachbeobachtungszeit zu versterben (HR 2,04, $95 \%$ Konfidenzintervall, 1,10-3,78; $p=0,02$ ) (Chow et al. 2008). In der 343 Patienten umfassenden prospektiven Beobachtungsstudie MACAS (Marburg Cardiomyopathy Study) 
wurde die Wertigkeit verschiedener nichtinvasiver Risikostratifikationsmethoden evaluiert. Die Haupteinschlusskriterien waren eine DCM und eine LVEF $\leq 45 \%$. Nach einer Beobachtungszeit von $52 \pm 21$ Monaten war die Bestimmung der LVEF der einzige Arrhythmieprädiktor mit signifikanter Wertigkeit bei Patienten mit Sinusrhythmus (95 \% Konfidenzintervall 1,5-3,3; $p=0,0001)$. 263 Patienten mit Sinusrhythmus absolvierten eine TWA-Testung (137 (52 \%) positive, 72 (27\%) negative und 54 (21\%) unbestimmbare Testungen). Es stellte sich heraus, dass eine positive TWA-Testung nicht mit einem signifikant höheren Risiko für lebensbedrohliche Arrhythmien einherging $(p=0,75)$. Nach multivariater Analyse konnte bei Patienten mit negativer TWA-Testung kein signifikant geringeres Risiko ermittelt werden, wohingegen nach univariater Analyse ein Trend erkennbar war $(p=0,13)$. Statistisch ähnlich verhielt es sich mit den unbestimmbaren TWA-Testungen. Hier ließ sich ein signifikant höheres Risiko für maligne Arrhythmien in der univariaten Analyse finden $(p=0,04)$. Auch hier war in der multivariaten Analyse keine Signifikanz zu ermitteln (Grimm et al. 2003).

\subsection{Anwendbarkeit von TWA-Testungen als Risikomarker für den SCD}

Mit der Entwicklung von Multikontaktelektroden und spezieller Software zur TWADetektion und Artefaktreduzierung gelang es, eine TWA-Testung nichtinvasiv im Rahmen einer Ergometrie durchzuführen (Albrecht et al. 1996). Dies stellte die technische Grundlage für die Etablierung der TWA-Testung in der nichtinvasiven Risikostratifikation lebensbedrohlicher Arrhythmien dar. Zahlreiche Studien konnten in verschiedenen Patientenpopulationen eine prognostische Wertigkeit, insbesondere einen hohen npW für die TWATestung, ermitteln (Myles et al. 2007). Trotz dieser guten Grundlage für eine Einführung als Risikomarker für den SCD wird die TWA-Testung in Deutschland vorwiegend in universitären Kardiologiezentren durchgeführt und meist ergänzend zu den üblichen Risikostratifikationsmethoden wie z. B. einer EPU. Im Folgenden werden mögliche Gründe diskutiert, inwiefern die technisch funktionierende TWA-Testung einen geeigneten Risikomarker darstellt.

Letztlich ist die prognostische Wertigkeit der TWA-Testung für verschiedene Patientengruppen noch nicht ausreichend bekannt, so dass man je nach Indikation für einen ICD präoperativ oft auf invasive Methoden nicht verzichten kann. Merchant et al. (2012) evaluierten fünf prospektive Studien, in denen mit insgesamt 2.883 Patienten eine TWATestung durchgeführt wurde. In dieser Untersuchung trugen Patienten mit einer LVEF $\leq 35 \%$ und negativer TWA-Testung ein niedrigeres Risiko für einen SCD als Patienten mit einem positiven $(p<0,001)$ oder unbestimmbaren Testergebnis $(p<0,001)$. Auch in der Patientengruppe mit einer LVEF $\geq 35 \%$ und positivem Testergebnis war die jährliche SCD-Rate signifikant höher als bei Patienten mit negativem bzw. unbestimmbarem Testergebnis ( $3,0 \%$ vs. jeweils $\left.0,3 \% ; p_{\text {negativ }}<0,001, p_{\text {unbestimmbar }}=0,003\right)$. Shizuta et al. veröffentlichten Anfang 2012 die prospektive PREVENT-SCD Studie mit 453 Patienten (LVEF $\leq 40 \%$ 
aufgrund ischämischer oder nichtischämischer Kardiomyopathie), in der bei 280 Patienten eine TWA-Testung durchgeführt werden konnte. Der Endpunkt wurde erreicht, sobald ein $S C D$, eine anhaltende VT bzw. ein VF auftraten oder eine appropriate Defibrillatortherapie ausgelöst wurde. Nach einer medianen Beobachtungszeit von drei Jahren erreichten signifikant weniger Patienten mit einem unauffälligen Testergebnis (97,0 \%) einen Studienendpunkt als Patienten mit auffälligem Ergebnis (89,5\%, $p=0,037)$. Shizuta et al. (2012) konnten damit der TWA-Testung bei Patienten mit einer linksventrikulären Dysfunktion einen hohen $\mathrm{npW}$ bezüglich lebensbedrohlichen ventrikulären Rhythmusstörungen zuschreiben. Im ersten Jahr betrug der npW $100 \%$, im zweiten Jahr 98,6\% und 97,0 \% im dritten Jahr. Gupta et al. (2012) führten eine Metaanalyse mit den von Januar 1990 bis Januar 2011 publizierten Arbeiten über die prognostische Wertigkeit der per Spektralanalyse ermittelten MTWA durch. Sie kamen schließlich zu dem Entschluss, dass trotz eines mäßigen Zusammenhanges zwischen Testergebnis und tachyarrhythmischen Ereignissen die Aussagekraft der Testung nicht ausreicht, um eine klinische Entscheidung substantiell zu beeinflussen. Für den Endpunkt „appropriate Schockabgabe“ konnte in der vorliegenden Arbeit bei den Patienten mit negativer TWA-Testung ein npW von $100 \%$ ermittelt werden, wenngleich kein signifikanter Unterschied gegenüber den Patienten mit nicht-negativem Befund gesehen wurde $(p=0,11)$, was wiederum durch eine zu geringe Fallzahl begründet sein könnte. Somit kann nach Auswertung der vorliegenden Daten die TWA-Testung als sehr vielversprechende Risikostratifikationsmethode betrachtet werden, jedoch kann eine Evidenz auf Basis der gewonnenen Methode nicht bewiesen werden. Die vorliegende und andere Arbeiten untersuchten letztlich, inwiefern die Ergebnisse einer nichtinvasiven TWA-Testung zur Risikostratifikation für lebensbedrohliche Herzrhythmusstörungen herangezogen werden können und ob eine Etablierung dieses Testverfahrens im klinischen Alltag möglich ist. Während Jackson et al. der Meinung sind, dass mit derzeit zur Verfügung stehenden Daten eine TWA-Testung nicht zur Identifizierung von Risikopatienten für lebensbedrohliche Herzrhythmusstörungen geeignet ist, sind Verrier et al. weiterhin der festen Überzeugung, bei der TWA-Testung handele es sich um eine verlässliche Methode in der Risikostratifikation (Jackson et al. 2012; Verrier et al. 2012). Folglich stimmt Verrier mit den ACC/AHA/ESC-Leitlinien überein, in der die Anwendung der TWA-Testung zur Risikostratifikation mit dem Evidenzgrad A und Indikationsklasse Ila bewertet wurde (Verrier et al. 2011; Zipes et al. 2006). Bei widersprüchlichen Konklusionen der vorangegangen Studien und ungewisser Wertigkeit der unbestimmbaren TWATestungen erscheint eine zeitnahe Reevaluation der Evidenzgrad- und Indikationsklasseneinteilung notwendig zu sein.

Die abschließende Frage dieser Arbeit galt dem Ausblick auf die Möglichkeiten der klinischen Anwendung von TWA-Testungen. Ein wesentlicher Nachteil dieses Testverfahrens ist seine eingeschränkte Anwendbarkeit. Etwa jeder dritte Patient, dessen Risiko für maligne Herzrhythmusstörungen stratifiziert werden soll, kann keiner TWA-Testung unterzogen werden. In der vorliegenden Arbeit mussten rund $32 \%$ der Patienten mit ICD- 
Erstimplantation von der Testung ausgeschlossen werden. Dieser Anteil ist vergleichbar mit dem in der von Kraaier et al. (2011) durchgeführten Studie zur Anwendbarkeit der TWA-Testung bei Patienten (ICM und DCM) vor primärpräventiver Implantation eines ICD. Patienten mit Vorhofflimmern können nicht mittels TWA-Testung untersucht werden. Bei etwa 30 bis $40 \%$ der Patienten mit einer Herzinsuffizienz lässt sich Vorhofflimmern ableiten (European Heart Rhythmus Association et al. 2010). Somit kann bei einer nicht zu vernachlässigenden Patientenanzahl keine TWA-Testung durchgeführt werden. Auch andere Risikostratifikationsmethoden wie die Herzfrequenzvaribiltätsmessung oder die Herzfrequenzturbulenzanalyse setzen einen Sinusrhythmus voraus. In dem hier untersuchten Kollektiv hatten von den ausgeschlossenen Patienten $39 \%$ keinen Sinusrhythmus. Für die TWA-Testung ist eine moderate Herzfrequenzsteigerung ähnlich wie bei einer Belastungsergometrie notwendig. Eine Reihe von Herzinsuffizienz-Patienten, deren Risiko eruiert werden soll, ist durch eine Gelenkerkrankung (z. B. Gonarthrose) in ihrer Bewegung stark eingeschränkt. Eine ergometrieähnliche Untersuchung ist dann häufig nicht durchführbar. Eine weitere Einschränkung in der Etablierung stellt die oben bereits diskutierte beeinflussende Wirkung von herzwirksamen Medikamenten dar. Aufgrund des sympatholytischen Effektes von Beta-Blockern erreichen viele Patienten mit einer Herzinsuffizienz nicht die für TWA-Testung erforderliche Herzfrequenz.

Die Ergebnisse dieser Arbeit und der anderer Arbeitsgruppen zeigen, dass auch nach fast 20 Jahren Forschung der Stellenwert der TWA-Testung im Rahmen der nichtinvasiven Risikostratifikation weiterhin zu diskutieren ist. Trotz erheblicher Kosten durch Verbrauchsmaterialien (Multikontaktelektroden) und sowohol hohen personellen als auch zeitlichen Aufwandes stellt die TWA-Testung aufgrund ihres nichtinvasiven Charakters ein attraktives Verfahren dar. Eine Fortführung oder eine Initiierung von weiteren Studien ist daher sehr wünschenswert. Dabei sollte keine Selektion durch die Indikation zur Implantation eines ICD oder durch die kardiale Grunderkrankung erfolgen, damit wie in dieser Arbeit das Patientenkollektiv dem klinischen Alltag entspricht. Die jüngste Studie zur nichtinvasiven SCD-Risikostratifikation ist die europäische EUTrigTreat-Studie von Seegers et al. (2012), deren Patientenrekrutierung (700 Patienten mit ICD) im Juli 2012 abgeschlossen werden konnte und deren Nachbeobachtungszeit im September 2014 endete. Auch während der Nachbeobachtung werden jährlich nichtinvasive Untersuchungen wie die TWA-Testung durchgeführt, um dann schließlich die offenen Fragen zur TWA-Testung und nichtinvasiven Risikostratifikation beantworten zu können. 


\section{Zusammenfassung}

In der Kardiologie hat die Risikostratifikation zur Bestimmung des individuellen Risikos für den plötzlichen Herztod (sudden cardiac death [SCD]) eine große Bedeutung. Sie ermöglicht die Identifizierung von Patienten, die gefährdet sind, eine lebensbedrohliche Herzrhythmusstörung zu erleiden. Die Risikostratifikation ist die entscheidende Grundlage, auf der die Indikationsstellung zur Implantation eines implantierbaren KardioverterDefibrillators (ICD) als primär- oder sekundärpräventive Maßnahme basiert. ICDs können hocheffektiv lebensbedrohliche Arrhythmien therapieren und so Patienten vor einem SCD bewahren.

Aktuell basiert die Risikostratifikation zum Teil noch auf invasiven Methoden wie zum Beispiel der elektrophysiologischen Untersuchung (EPU). Die Bedeutung von neuen nichtinvasiven Methoden zur Risikostratifikation ist Gegenstand aktueller Forschung. Seit etwa zwei Jahrzehnten wird die T-Wellen-Alternans-Testung (TWA-Testung) kontrovers diskutiert. Die TWA-Testung erfasst das herzfrequenzabhängige Alternieren der T-Welle von Herzschlag-zu-Herzschlag im Mikrovoltbereich im Rahmen einer ergometrieähnlichen Untersuchung mit moderater Belastung.

Ziel dieser prospektiven Arbeit war es, die prognostische Wertigkeit der TWA-Testung für das Auftreten lebensbedrohlicher ventrikulärer Arrhythmien bei einem weitestgehend unselektierten Patientenkollektiv zu untersuchen. Dazu wurden insgesamt 134 Patienten des Herzzentrums Göttingen prospektiv in eine monozentrische Beobachtungsstudie aufgenommen, bei denen vor ICD-Erstimplantation eine TWA-Testung durchgeführt wurde. Als Endpunkte wurden appropriate ICD-Schockabgaben und die Gesamtmortalität vordefiniert. Die mittlere Nachbeobachtungszeit betrug zwölf Monate. Während dieser erreichten elf Patienten einen Endpunkt. Es konnten drei appropriate Schockabgaben registriert werden. Acht Patienten verstarben. Innerhalb des beobachteten Kollektivs hatten Patienten mit einer unbestimmbaren TWA-Testung ein signifikant höheres Risiko, einen der beiden Endpunkte zu erreichen als Patienten mit negativem Testergebnis. Es bestand keine signifikant unterschiedliche Ereignisrate zwischen den Patientengruppen mit positivem oder negativem Testergebnis.

Für die Bestimmung der prognostischen Wertigkeit der TWA-Testung bedarf es weiterer klinischer Untersuchungen mit größeren Patientenpopulationen und längerer Beobachtungszeit. 


\section{Anhang}

\subsection{Positionen aller 14 Elektroden}

\begin{tabular}{|c|c|c|}
\hline Ableitung & Elektrodenposition & Art der Elektrode \\
\hline RA & unterhalb der rechten Clavicula & Silber/Silberchlorid-Elektrode \\
\hline LA & unterhalb der linken Clavicula & Silber/Silberchlorid-Elektrode \\
\hline $\mathrm{RL}$ & $\begin{array}{l}\text { auf Höhe der letzten echten Rippe rechts, } \\
\text { auf Höhe von RA }\end{array}$ & Silber/Silberchlorid-Elektrode \\
\hline LL & $\begin{array}{l}\text { auf Höhe der letzten echten Rippe links, } \\
\text { auf Höhe von LA }\end{array}$ & Silber/Silberchlorid-Elektrode \\
\hline $\mathrm{V}_{1}$ & vierter Interkostalraum rechts parasternal & Silber/Silberchlorid-Elektrode \\
\hline$V_{2}$ & vierter Interkostalraum links parasternal & Multikontaktelektrode \\
\hline$V_{3}$ & zwischen $V_{2}$ und $V_{4}$ auf der fünftem Rippe & Silber/Silberchlorid-Elektrode \\
\hline $\mathrm{V}_{4}$ & $\begin{array}{l}\text { Medioclavikularlinie links, über fünften Interkostal- } \\
\text { raum }\end{array}$ & Multikontaktelektrode \\
\hline$V_{5}$ & gleiche Höhe wie $V_{4}$, auf der vorderen Axillarlinie & Multikontaktelektrode \\
\hline $\mathrm{V}_{6}$ & gleiche Höhe wie $V_{4}$, auf der mittleren Axillarlinie & Multikontaktelektrode \\
\hline 1 & mittlere Axillarlinie, über fünftem Interkostalraum & Multikontaktelektrode \\
\hline $\mathrm{E}$ & Xiphoid, auf der vorderen Medianlinie & Multikontaktelektrode \\
\hline M & sagittal gegenüber von $V_{2}$ auf dem Rücken & Silber/Silberchlorid-Elektrode \\
\hline $\mathrm{H}$ & Manubrium sterni & Multikontaktelektrode \\
\hline
\end{tabular}

Tab. 6 Elektrodenpositionen aller 14 Elektroden 


\section{Literaturverzeichnis}

Adachi K, Ohnishi Y, Yokoyama M (2001): Risk stratification for sudden cardiac death in dilated cardiomyopathy using microvolt-level T-wave alternans. Jpn Circ J 65 (2): 7680 .

Albrecht P, Arnold J, Krishnamachari S, Cohen RJ (1996): Exercise recordings for the detection of T wave alternans. Promises and pitfalls. J Electrocardiol (29): 46-51.

Andresen D (2007): Epidemiologie des plötzlichen Herztodes. Wer ist gefährdet? Intensivmed 44 (4): 188-193.

Armoundas AA, Nanke T, Cohen RJ (2000): Images in cardiovascular medicine. T-wave alternans preceding torsade de pointes ventricular tachycardia. Circulation 101(21): 2550.

Armoundas AA, Tomaselli GF, Esperer HD (2002): Pathophysiological basis and clinical application of T-wave alternans. J Am Coll Cardiol 40(2): 207-217.

Baddour LM, Epstein AE, Erickson CC, Knight BP, Levison ME, Lockhart PB, Masoudi FA, Okum EJ, Wilson WR, Beerman LB et al. (2010): Update on cardiovascular implantable electronic device infections and their management: a scientific statement from the American Heart Association. Circulation 121(3): 458-477.

Bardy GH, Lee KL, Mark DB, Poole JE, Packer DL, Boineau R, Domanski M, Troutman C, Anderson J, Johnson $\mathrm{G}$ et al. (2005): Amiodarone or an implantable cardioverterdefibrillator for congestive heart failure. N Engl J Med 352(3): 225-237.

Baravelli M, Salerno-Uriarte D, Guzzetti D, Rossi MC, Zoli L, Forzani T, Salerno-Uriarte JA (2005): Predictive significance for sudden death of microvolt-level T wave alternans in New York Heart Association class II congestive heart failure patients: a prospective study. Int J Cardiol 105(1): 53-57.

Barthel P, Schneider R, Bauer A, Ulm K, Schmitt C, Schömig A, Schmidt G (2003): Risk stratification after acute myocardial infarction by heart rate turbulence. Circulation 108(10): 1221-1226.

Bayés de Luna A, Coumel P, Leclercq JF (1989): Ambulatory sudden cardiac death: mechanisms of production of fatal arrhythmia on the basis of data from 157 cases. Am Heart J 117(1): 151-159.

Benninghoven D, Kunzendorf S, Heberlein I, Jantscheck G (2006): Angststörungen bei Patienten mit implantiertem Kardioverter-Defibrillator (ICD). Psychotherapeut $\underline{51}(3)$ : 206-213.

Bloomfield DM, Hohnloser SH, Cohen RJ (2002): Interpretation and classification of microvolt T wave alternans tests. J Cardiovasc Electrophysiol 13(5): 502-512 
Bloomfield DM, Steinman RC, Namerow PB, Parides M, Davidenko J, Kaufman ES, Shinn T, Curtis A, Fontaine J, Holmes D, et al. (2004): Microvolt T-wave alternans distinguishes between patients likely and patients not likely to benefit from implanted cardiac defibrillator therapy: a solution to the Multicenter Automatic Defibrillator Implantation Trial (MADIT) II conundrum. Circulation 110(14): 1885-1889.

Bloomfield DM, Bigger JT, Steinman RC, Namerow PB, Parides MK, Curtis AB, Kaufman ES, Davidenko JM, Shinn TS, Fontaine JM (2006): Microvolt T-wave alternans and the risk of death or sustained ventricular arrhythmias in patients with left ventricular dysfunction. J Am Coll Cardiol 477(2): 456-463.

Bode-Schnurbus L, Bocker D, Block M, Gradaus R, Heinecke A, Breithardt G, Borggrefe M (2003): QRS duration: a simple marker for predicting cardiac mortality in ICD patients with heart failure. Heart $\underline{89}(10)$ : 1157-1162.

Borne RT, Varosy PD, Masoudi FA (2013): Implantable Cardioverter-Defibrillator Shocks: Epidemiology, Outcomes, and Therapeutic Approaches. JAMA Intern Med 173(10): 859-865.

Buxton AE (2005): Should everyone with an ejection fraction less than or equal to $30 \%$ receive an implantable cardioverter-defibrillator? Not everyone with an ejection fraction $<$ or $=30 \%$ should receive an implantable cardioverter-defibrillator. Circulation 111(19): 2537-2549.

Buxton AE, Lee KL, Fisher JD, Josephson ME, Prystowsky EN, Hafley G (1999): A randomized study of the prevention of sudden death in patients with coronary artery disease. Multicenter Unsustained Tachycardia Trial Investigators. N Engl J Med 341(25): 1882-1890.

Buxton AE, Lee KL, Hafley GE, Wyse DG, Fisher JD, Lehmann MH, Pires LA, Gold MR, Packer $\mathrm{DL}$, Josephson ME, et al. (2002): Relation of ejection fraction and inducible ventricular tachycardia to mode of death in patients with coronary artery disease: an analysis of patients enrolled in the multicenter unsustained tachycardia trial. Circulation 106(19): 2466-2472.

Calò L, De Santo T, Nuccio F, Sciarra L, De Luca L, Stefano LM, Piroli E, Zuccaro L, Rebecchi $M$, de Ruvo $E$ et al. (2011): Predictive value of microvolt T-wave alternans for cardiac death or ventricular tachyarrhythmic events in ischemic and nonischemic cardiomyopathy patients: a meta-analysis. Ann Noninvasive Electrocardiol 16(4): 388402.

Chan PS, Bartone C, Booth T, Kereiakes D, Chow T (2007): Prognostic implication of redefining indeterminate microvolt T-wave alternans studies as abnormal or normal. Am Heart J 153 (4): 523-529.

Chow T, Kereiakes DJ, Bartone C, Booth T, Schloss EJ, Waller T, Chung ES, Menon S, Nallamothu BK, Chan PS (2006): Prognostic utility of microvolt T-wave alternans in 
risk stratification of patients with ischemic cardiomyopathy. J Am Coll Cardiol 47(9): 1820-1827.

Chow T, Kereiakes DJ, Bartone C, Booth T, Schloss EJ, Waller T, Chung E, Menon S, Nallamothu BK, Chan PS (2007): Microvolt T-wave alternans identifies patients with ischemic cardiomyopathy who benefit from implantable cardioverterdefibrillator therapy. J Am Coll Cardiol 49 (1): 50-58.

Chow T, Kereiakes DJ, Onufer J, Woelfel A, Gursoy S, Peterson BJ, Brown ML, Pu W, Benditt DG (2008): Does microvolt T-wave alternans testing predict ventricular tachyarrhythmias in patients with ischemic cardiomyopathy and prophylactic defibrillators? The MASTER (Microvolt T Wave Alternans Testing for Risk Stratification of Post-Myocardial Infarction Patients) trial. J Am Coll Cardiol 52(20): 1607-1615.

Connolly SJ, Gent M, Roberts RS, Dorian P, Roy D, Sheldon RS, Mitchell LB, Green MS, Klein GJ, O'Brien B (2000a): Canadian implantable defibrillator study (CIDS) : a randomized trial of the implantable cardioverter defibrillator against amiodarone. Circulation 101(11): 1297-1302.

Connolly SJ, Hallstrom AP, Cappato R, Schron EB, Kuck KH, Zipes DP, Greene HL, Boczor S, Domanski M, Follmann D, et al. (2000b): Meta-analysis of the implantable cardioverter defibrillator secondary prevention trials. AVID, CASH and CIDS studies. Antiarrhythmics vs Implantable Defibrillator study. Cardiac Arrest Study Hamburg . Canadian Implantable Defibrillator Study. Eur Heart J 21(24): 2071-2078.

Costantini O, Hohnloser SH, Kirk MM, Lerman BB, Baker JH, 2nd, Sethuraman B, Dettmer MM, Rosenbaum DS (2009): The ABCD (Alternans Before Cardioverter Defibrillator) Trial: strategies using T-wave alternans to improve efficiency of sudden cardiac death prevention. J Am Coll Cardiol 53 (6): 471-479.

Cutler MJ, Rosenbaum DS (2009): Explaining the clinical manifestations of T wave alternans in patients at risk for sudden cardiac death. Heart Rhythm $\underline{6}$ (3): 22-28.

Daniłowicz-Szymanowicz L, Szwoch M, Raczak J, Raczak G (2012): Negative result of microvolt T-wave alternans test is helpful in scheduling the order of cardioverterdefibrillator implantation in primary prevention of sudden cardiac death in individuals with the left ventricular systolic dysfunction. Pol Merkur Lekarski 33(196): 193197.

Daubert JP, Zareba W, Cannom DS, McNitt S, Rosero SZ, Wang P, Schuger C, Steinberg JS, Higgins SL, Wilber DJ, et al. (2008): Inappropriate implantable cardioverterdefibrillator shocks in MADIT II: frequency, mechanisms, predictors, and survival impact. J Am Coll Cardiol 51(14): 1357-1365.

Eber B, Porodko M, Auer J, Berent R, Weber T, Mayr, Maurer E (2000): Betablocker-update 2000. J Kardiol $\underline{7}(11)$ : 474-481. 
European Heart Rhythm Association; European Association for Cardio-Thoracic Surgery, Camm AJ, Kirchhof P, Lip GY, Schotten U, Savelieva I, Ernst S, Van Gelder IC, AlAttar N (2010): Guidelines for the management of atrial fibrillation: the Task Force for the Management of Atrial Fibrillation of the European Society of Cardiology (ESC). Eur Heart J 31(19): 2369-429.

Frank E (1956): An accurate, clinically practical system for spatial vectorcardiography. Circulation 13(5): 737-749.

Gehi AK, Stein RH, Metz LD, Gomes JA (2005): Microvolt T-wave alternans for the risk stratification of ventricular tachyarrhythmic events: a meta-analysis. J Am Coll Cardiol $\underline{46}(1):$ 75-82.

Germano JJ, Reynolds M, Essebag V, Josephson ME (2006): Frequency and causes of implantable cardioverter-defibrillator therapies: is device therapy proarrhythmic? Am J Cardiol 97(8): 1255-1261.

Gold MR, Bloomfield DM, Anderson KP, El-Sherif NE, Wilber DJ, Groh WJ, Estes NA, 3rd, Kaufman ES, Greenberg ML, Rosenbaum DS (2000): A comparison of T-wave alternans, signal averaged electrocardiography and programmed ventricular stimulation for arrhythmia risk stratification. J Am Coll Cardiol 36(7): 2247-2253.

Gold MR, Ip JH, Costantini O, Poole JE, McNulty S, Mark DB, Lee KL, Bardy GH (2008): Role of microvolt T-wave alternans in assessment of arrhythmia vulnerability among patients with heart failure and systolic dysfunction: primary results from the T-wave alternans sudden cardiac death in heart failure trial substudy. Circulation 118(20): 2022-2028.

Goldberger JJ, Cain ME, Hohnloser SH, Kadish AH, Knight BP, Lauer MS, Maron BJ, Page RL, Passman RS, Siscovick D, et al. (2008): American Heart Association/American College of Cardiology Foundation/Heart Rhythm Society scientific statement on noninvasive risk stratification techniques for identifying patients at risk for sudden cardiac death: a scientific statement from the American Heart Association Council on Clinical Cardiology Committee on Electrocardiography and Arrhythmias and Council on Epidemiology and Prevention. Circulation 118(14): 1497-1518.

Greene HL (1990): Sudden arrhythmic cardiac death-mechanisms, resuscitation and classification: the Seattle perspective. Am J Cardiol $\underline{65}$ (4): 4-12.

Grimm W, Christ M, Bach J, Müller HH, Maisch B (2003): Noninvasive arrhythmia risk stratification in idiopathic dilated cardiomyopathy: results of the Marburg Cardiomyopathy Study. Circulation 108(23): 2883-2891.

Grimm W, Plachta E, Maisch B (2006): Antitachycardia pacing for spontaneous rapid ventricular tachycardia in patients with prophylactic cardioverter-defibrillator therapy. Pacing Clin Electrophysiol 29(7): 759-764. 
Gupta A, Hoang DD, Karliner L, Tice JA, Heidenreich P, Wang PJ, Turakhia MP Ability of microvolt T-wave alternans to modify risk assessment of ventricular tachyarrhythmic events: a meta-analysis. Am Heart J 163(3): 354-364.

Hering HE (1909): Experimentelle Studien an Säugethieren über das Elektrocardiogram. Zschr Exper Path Therapie 7: 363.

Hohnloser SH, Klingenheben T, Zabel M, Li YG, Albrecht P, Cohen RJ (1997): T wave alternans during exercise and atrial pacing in humans. J Cardiovasc Electrophysiol $\underline{8}(9)$ : 987-993.

Hohnloser SH, Klingenheben T, Li YG, Zabel M, Peetermans J, Cohen RJ (1998): T wave alternans as a predictor of recurrent ventricular tachyarrhythmias in ICD recipients: prospective comparison with conventional risk markers. J Cardiovasc Electrophysiol $\underline{9}(12): 1258-1268$.

Hohnloser SH, Klingenheben T, Bloomfield D, Dabbous O, Cohen RJ (2003): Usefulness of microvolt T-wave alternans for prediction of ventricular tachyarrhythmic events in patients with dilated cardiomyopathy: results from a prospective observational study. J Am Coll Cardiol 41 (12): 2220-2224.

Huikuri HV, Castellanos A, Myerburg RJ (2001): Sudden death due to cardiac arrhythmias. N Engl J Med 345(20): 1473-1482.

Ikeda T, Sakata T, Takami M, Kondo N, Tezuka N, Nakae T, Noro M, Enjoji Y, Abe R, Sugi K, et al. (2000): Combined assessment of T-wave alternans and late potentials used to predict arrhythmic events after myocardial infarction. A prospective study. J Am Coll Cardiol 35(3): 722-730.

Ikeda T, Yoshino H, Sugi K, Tanno K, Shimizu H, Watanabe J, Kasamaki Y, Yoshida A, Kato T (2006): Predictive value of microvolt T-wave alternans for sudden cardiac death in patients with preserved cardiac function after acute myocardial infarction: results of a collaborative cohort study. J Am Coll Cardiol 48(11): 2268-2274.

Jackson CE, Myles RC, Cobbe SM, Petrie MC, McMurray JJ (2012): Microvolt T-wave alternans testing has no role at present in guiding therapy for patients at high risk of ventricular arrhythmias. J Am Coll Cardiol $\underline{59}(9): 856$.

Jung W, Andresen D, Block M, Böcker D, Hohnloser SH, Kuck KH, Sperzel J (2006): Leitlinien zur Implantation von Defibrillatoren. Clin Res Cardiol 95(12): 696-708.

Kaufman ES, Bloomfield DM, Steinman RC, Namerow PB, Costantini O, Cohen RJ, Bigger JT, Jr. (2006): "Indeterminate" microvolt T-wave alternans tests predict high risk of death or sustained ventricular arrhythmias in patients with left ventricular dysfunction. J Am Coll Cardiol 48 (7): 1399-1404. 
Kavesh NG, Shorofsky SR, Sarang SE, Gold MR (1998): Effect of heart rate on T wave alternans. J Cardiovasc Electrophysiol $\underline{9}(7):$ 703-708.

Klingenheben T, Siedow A, Credner SC, Gronefeld G, Zabel M, Li YG, Hohnloser SH (1999): T-wave alternans in microwave frequency as a new indicator of disordered ventricular repolarization: pathophysiology, methodology, clinical results. Z Kardiol 88(12): 974-981.

Klingenheben T, Credner S, Li YG, Bender B, Hohnloser SH (2000): Mikrovolt-T-WellenAlternans - ein neuer Marker zur nichtinvasiven Risikostratifikation. Z Kardiol 899(3): 57-61.

Klingenheben T, Gronefeld G, Li YG, Hohnloser SH (2001): Effect of metoprolol and d,Isotalol on microvolt-level T-wave alternans. Results of a prospective, double-blind, randomized study. J Am Coll Cardiol 38(7): 2013-2019.

Koller MT, Schaer B, Wolbers M, Sticherling C, Bucher HC, Osswald S (2008): Death without prior appropriate implantable cardioverter-defibrillator therapy: a competing risk study. Circulation 117(15): 1918-1926.

Koneru JN, Swerdlow CD, Wood MA, Ellenbogen KA (2011): Minimizing inappropriate or "unnecessary" implantable cardioverter-defibrillator shocks: appropriate programming. Circ Arrhythm Electrophysiol 4⑸: 778-790.

Kraaier K, McCracken T, van der Palen J, Wilde AA, Scholten MF (2011): Is T-wave alternans testing feasible in candidates for prophylactic implantable defibrillators? Neth Heart J 19(1): 6-9.

Kuck KH, Cappato R, Siebels J, Ruppel R (2000): Randomized comparison of antiarrhythmic drug therapy with implantable defibrillators in patients resuscitated from cardiac arrest : the Cardiac Arrest Study Hamburg (CASH). Circulation 102(7): 748-754.

La Rovere MT, Bigger JT, Jr., Marcus FI, Mortara A, Schwartz PJ (1998): Baroreflex sensitivity and heart-rate variability in prediction of total cardiac mortality after myocardial infarction. ATRAMI (Autonomic Tone and Reflexes After Myocardial Infarction) Investigators. Lancet $\underline{351}(9101)$ : 478-484.

La Rovere MT, Pinna GD, Hohnloser SH, Marcus FI, Mortara A, Nohara R, Bigger JT, Jr., Camm AJ, Schwartz PJ (2001): Baroreflex sensitivity and heart rate variability in the identification of patients at risk for life-threatening arrhythmias: implications for clinical trials. Circulation 103(16): 2072-2077.

Ladwig KH, Ronel J, Baumert J, Kolb C (2010): Psychological comorbidity and quality of life in patients with an implantable cardioverter/defribrillator (ICD). Herzschrittmacherther Elektrophysiol 21(2): 129-136. 
Larsen GK, Evans J, Lambert WE, Chen Y, Raitt MH (2011): Shocks burden and increased mortality in implantable cardioverter-defibrillator patients. Heart Rhythm $\underline{8}(12)$ : 1881-1886.

Lewandowski M, Kossuth I, Zielonka J, Wielusiński M, Kazimierczak A, Kornacewicz Jach Z, Przybycień K, Kaliszczak R (2011): Microvolt T-wave alternans for the risk stratification of dangerous ventricular arrhythmias in patients with previously implanted automatic cardioverter-defibrillator. Kardiol Pol 69 (6): 580-585.

Lüderitz B (2002): We have come a long way with device therapy: historical perspectives on antiarrhythmic electrotherapy. J Cardiovasc Electrophysiol 13 (1): 2-8.

Maisel WH (2007): Transvenous implantable cardioverter-defibrillator leads: the weakest link. Circulation 115(19): 2461-2463.

Martin DT, Shoraki A, Nesto RW, Rutter MK (2009): Influence of diabetes and/or myocardial infarction on prevalence of abnormal T-wave alternans. Ann Noninvasive Electrocardiol 14(4): 355-359.

Merchant FM, Ikeda T, Pedretti RF, Salerno-Uriarte JA, Chow T, Chan PS, Bartone C, Hohnloser SH, Cohen RJ, Armoundas AA (2012): Clinical utility of microvolt T-wave alternans testing in identifying patients at high or low risk of sudden cardiac death. Heart Rhythm 9 (8): 1256-1264.

Mirowski M, Reid PR, Mower MM, Watkins L, Gott VL, Schauble JF, Langer A, Heilman MS, Kolenik SA, Fischell RE, et al. (1980): Termination of malignant ventricular arrhythmias with an implanted automatic defibrillator in human beings. $N$ Engl J Med 303(6): 322-324.

Miyazaki S, Shah AJ, Haissaguerre M (2010): Early repolarization syndrome - a new electrical disorder associated with sudden cardiac death -. Circ J 74(10): 2039-2044.

Moss AJ (1997): A comparison of antiarrhythmic-drug therapy with implantable defibrillators in patients resuscitated from near-fatal ventricular arrhythmias. The Antiarrhythmics versus Implantable Defibrillators (AVID) Investigators. $N$ Engl J Med 337(22): 1576-1583.

Moss AJ, Hall WJ, Cannom DS, Daubert JP, Higgins SL, Klein H, Levine JH, Saksena S, Waldo AL, Wilber D, et al. (1996): Improved survival with an implanted defibrillator in patients with coronary disease at high risk for ventricular arrhythmia. Multicenter Automatic Defibrillator Implantation Trial Investigators. N Engl J Med 335(26): 19331940.

Moss AJ, Zareba W, Hall WJ, Klein H, Wilber DJ, Cannom DS, Daubert JP, Higgins SL, Brown MW, Andrews ML (2002): Prophylactic implantation of a defibrillator in patients with myocardial infarction and reduced ejection fraction. N Engl J Med 346(12): 877-883. 
Moss AJ, Schuger C, Beck CA, Brown MW, Cannom DS, Daubert JP, Estes NA 3rd, Greenberg $\mathrm{H}$, Hall WJ, Huang DT, et al. (2012): Reduction in inappropriate therapy and mortality through ICD programming. N Engl J Med 367(24): 2275-2283.

Myerburg RJ, Mitrani R, Interian A, Jr., Castellanos A (1998): Interpretation of outcomes of antiarrhythmic clinical trials: design features and population impact. Circulation 97(15): 1514-1521.

Myerburg RJ, Castellanos A (2006): Emerging paradigms of the epidemiology and demographics of sudden cardiac arrest. Heart Rhythm $\underline{3}(2): 235-239$.

Myles RC, Jackson CE, Tsorlalis I, Petrie MC, McMurray JJ, Cobbe SM (2007): Is microvolt Twave alternans the answer to risk stratification in heart failure? Circulation 116(25): 2984-2991.

Narayan SM (2006): T-wave alternans and the susceptibility to ventricular arrhythmias. J Am Coll Cardiol 47(2): 269-281.

Occhetta E, Bortnik M, Magnani A, Francalacci G, Marino P (2006): Inappropriate implantable cardioverter-defibrillator discharges unrelated to supraventricular tachyarrhythmias. Europace $\underline{8}(10):$ 863-869.

Okin PM, Devereux RB, Howard BV, Fabsitz RR, Lee ET, Welty TK (2000): Assessment of QT interval and QT dispersion for prediction of all-cause and cardiovascular mortality in American Indians: The Strong Heart Study. Circulation 101(1): 61-66.

Oshodi GO, Wilson LD, Costantini O, Rosenbaum DS (2008): Microvolt T wave alternans: Mechanisms and implications for prediction of sudden cardiac death; in: Electrical diseases of the heart: Genetics, mechanisms, treatment, prevention; herausgegeben von Gussak I, Antzelevitch C ; Springer-Verlag, 394-408.

Pandit SV (2010): T-wave alternans testing: can it predict arrhythmogenesis? Heart Rhythm ㄱ(6): 769-770.

Pastore JM, Girouard SD, Laurita KR, Akar FG, Rosenbaum DS (1999): Mechanism linking Twave alternans to the genesis of cardiac fibrillation. Circulation 99(10): 1385-1394.

Perkiömäki JS, Bloch Thomsen PE, Kiviniemi AM, Messier MD, Huikuri HV (2011): Risk factors of self-terminating and perpetuating ventricular tachyarrhythmias in postinfarction patients with moderately depressed left ventricular function, a CARISMA sub-analysis. Europace 13(11): 1604-1611.

Platt SB, Vijgen JM, Albrecht P, Van Hare GF, Carlson MD, Rosenbaum DS (1996): Occult T wave alternans in long QT syndrome. J Cardiovasc Electrophysiol 7(2): 144-148.

Poole JE, Johnson GW, Hellkamp AS, Anderson J, Callans DJ, Raitt MH, Reddy RK, Marchlinski FE, Yee R, Guarnieri T, et al. (2008): Prognostic importance of defibrillator shocks in patients with heart failure. N Engl J Med 359(10): 1009-1017. 
Rashba EJ, Cooklin M, MacMurdy K, Kavesh N, Kirk M, Sarang S, Peters RW, Shorofsky SR, Gold MR (2002): Effects of selective autonomic blockade on T-wave alternans in humans. Circulation 105(7): 837-842.

Rashba EJ, Osman AF, Macmurdy K, Kirk MM, Sarang SE, Peters RW, Shorofsky SR, Gold MR (2004): Enhanced detection of arrhythmia vulnerability using $T$ wave alternans, left ventricular ejection fraction, and programmed ventricular stimulation: a prospective study in subjects with chronic ischemic heart disease. J Cardiovasc Electrophysiol $\underline{15}(2)$ : 170-176.

Rashba EJ, Cooklin M, MacMurdy K, Kavesh N, Kirk M, Sarang S, Peters RW, Shorofsky SR, Gold MR (2002): Effects of selective autonomic blockade on T-wave alternans in humans. Circulation 105(7): 837-842.

Richter S, Duray G, Hohnloser SH (2005): How to analyze T-wave alternans. Heart Rhythm 2(11): 1268-1271.

Rosenbaum DS (2001): T wave alternans: a mechanism of arrhythmogenesis comes of age after 100 years. J Cardiovasc Electrophysiol 12(2): 207-209.

Rosenbaum DS, Jackson LE, Smith JM, Garan H, Ruskin JN, Cohen RJ (1994): Electrical alternans and vulnerability to ventricular arrhythmias. N Engl J Med 330(4): 235-241.

Rosenbaum DS, Albrecht P, Cohen RJ (1996): Predicting sudden cardiac death from T wave alternans of the surface electrocardiogram: promise and pitfalls. $J$ Cardiovasc Electrophysiol $\underline{7}(11):$ 1095-1111.

Salerno-Uriarte JA, De Ferrari GM, Klersy C, Pedretti RF, Tritto M, Sallusti L, Libero L, Pettinati G, Molon G, Curnis A et al. (2007): Prognostic value of T-wave alternans in patients with heart failure due to nonischemic cardiomyopathy: results of the ALPHA Study. J Am Coll Cardiol 50(19): 1896-1904.

Schmitt C, Montero M, Melichercik J (1994): Significance of supraventricular tachyarrhythmias in patients with implanted pacing cardioverter defibrillators. Pacing Clin Electrophysiol 17(3 Pt 1): 295-302.

Schoels W, Steinhaus D, Johnson WB, O'Hara G, Schwab JO, Jenniskens I, Degroot PJ, Tang F, Helmling E (2007): Optimizing implantable cardioverter-defibrillator treatment of rapid ventricular tachycardia: antitachycardia pacing therapy during charging. Heart Rhythm 4(7): 879-885.

Schron EB, Exner DV, Yao Q, Jenkins LS, Steinberg JS, Cook JR, Kutalek SP, Friedman PL, Bubien RS, Page RL, et al. (2002): Quality of life in the antiarrhythmics versus implantable defibrillators trial: impact of therapy and influence of adverse symptoms and defibrillator shocks. Circulation 105(5): 589-594. 
Schuster HP Trappe HJ: EKG-Kurs für Isabel. 4. überarbeitete und erweiterte Auflage; Georg Thieme Verlag, Stuttgart 2005, 14.

Schwartz PJ, Malliani A (1975): Electrical alternation of the T-wave: clinical and experimental evidence of its relationship with the sympathetic nervous system and with the long Q-T syndrome. Am Heart J 89(1): 45-50.

Secemsky EA, Verrier RL, Cooke G, Ghossein C, Subacius H, Manuchehry A, Herzog CA, Passman R (2011): High prevalence of cardiac autonomic dysfunction and T-wave alternans in dialysis patients. Heart Rhythm $\underline{8}(4): 592-598$.

Seegers J, Zabel M, Lüthje L, Vollmann D (2010): Ventricular oversensing due to manufacturer-related differences in implantable cardioverter-defibrillator signal processing and sensing lead properties. Europace 12(10): 1460-1466.

Seegers J, Vos MA, Flevari P, Willems R, Sohns C, Vollmann D, Lüthje L, Kremastinos DT, Floré $\mathrm{V}$, Meine $\mathrm{M}$ et al. (2012): Rationale, objectives, and design of the EUTrigTreat clinical study: a prospective observational study for arrhythmia risk stratification and assessment of interrelationships among repolarization markers and genotype. Europace 14(3): 416-422.

Sha J, Zhang S, Tang M, Chen K, Zhao X, Wang F (2011): Fragmented QRS is associated with all-cause mortality and ventricular arrhythmias in patient with idiopathic dilated cardiomyopathy. Ann Noninvasive Electrocardiol 16(3): 270-275.

Shizuta S, Ando K, Nobuyoshi M, Ikeda T, Yoshino H, Hiramatsu S, Kazatani Y, Yamashiro K, Okajima K, Kajiya T et al. (2012): Prognostic utility of T-wave alternans in a realworld population of patients with left ventricular dysfunction: the PREVENT-SCD study. Clin Res Cardiol 101 (2): 89-99.

Smith JM, Clancy EA, Valeri CR, Ruskin JN, Cohen RJ (1988): Electrical alternans and cardiac electrical instability. Circulation 77(1): 110-121.

Sohail MR, Hussain S, Le KY, Dib C, Lohse CM, Friedman PA, Hayes DL, Uslan DZ, Wilson WR, Steckelberg JM et al. (2011): Risk factors associated with early- versus lateonset implantable cardioverter-defibrillator infections. J Interv Card Electrophysiol

Stein PK, Barzilay JI (2011): Relationship of abnormal heart rate turbulence and elevated CRP to cardiac mortality in low, intermediate, and high-risk older adults. J Cardiovasc Electrophysiol 22(2): 122-127.

Sweeney MO, Wathen MS, Volosin K, Abdalla I, DeGroot PJ, Otterness MF, Stark AJ (2005): Appropriate and inappropriate ventricular therapies, quality of life, and mortality among primary and secondary prevention implantable cardioverter defibrillator patients: results from the Pacing Fast VT REduces Shock ThErapies (PainFREE Rx II) trial. Circulation 111(22): 2898-2905. 
Tapanainen JM, Still AM, Airaksinen KE, Huikuri HV (2001): Prognostic significance of risk stratifiers of mortality, including $\mathrm{T}$ wave alternans, after acute myocardial infarction: results of a prospective follow-up study. J Cardiovasc Electrophysiol 12(6): 645-652.

Tebbenjohanns J, Willems S, Antz M, Pfeiffer D, Seidl KH, Lewalter T (2008): Kommentar zu den „ACC/AHA/ESC 2006 guidelines for management of patients with ventricular arrhythmias and the prevention of sudden cardiac death - executive summary". Der Kardiologe 2(5): 363-388.

van Rees JB, Borleffs CJ, de Bie MK, Stijnen T, van Erven L, Bax JJ, Schalij MJ (2011): Inappropriate implantable cardioverter-defibrillator shocks: incidence, predictors, and impact on mortality. J Am Coll Cardio. 57(5): 556-562.

van Welsenes GH, van Rees JB, Borleffs CJ, Cannegieter SC, Bax JJ, van Erven L, Schalij MJ (2011): Long-term follow-up of primary and secondary prevention implantable cardioverter defibrillator patients. Europace 13(3): 389-394.

Verrier RL, Nearing BD, Kwaku KF (2005): Noninvasive sudden death risk stratification by ambulatory ECG-based T-wave alternans analysis: evidence and methodological guidelines. Ann Noninvasive Electrocardiol 10(1): 110-120.

Verrier RL, Kumar K, Nearing BD (2009): Basis for sudden cardiac death prediction by Twave alternans from an integrative physiology perspective. Heart Rhythm $\underline{6}(3)$ : 416-422.

Verrier RL, Klingenheben T, Malik M, El-Sherif N, Exner DV, Hohnloser SH, Ikeda T, Martínez JP, Narayan SM, Nieminen T, et al. (2011): Microvolt T-wave alternans physiological basis, methods of measurement, and clinical utility--consensus guideline by International Society for Holter and Noninvasive Electrocardiology. J Am Coll Cardiol 58(13): 1309-1324.

Verrier RL, Klingenheben T, Malik M, El-Sherif N, Exner DV, Hohnloser SH, Ikeda T, Martínez JP, Narayan SM, Nieminen T (2012): Microvolt T-wave alternans testing has a role in arrhythmia risk stratification. J Am Coll Cardiol 59(17): 1572-1573.

Vollmann D, Luthje L, Vonhof S, Unterberg C (2005): Inappropriate therapy and fatal proarrhythmia by an implantable cardioverter-defibrillator. Heart Rhythm 2(3): 307-309.

Wathen MS, Sweeney MO, DeGroot PJ, Stark AJ, Koehler JL, Chisner MB, Machado C, Adkisson WO (2001): Shock reduction using antitachycardia pacing for spontaneous rapid ventricular tachycardia in patients with coronary artery disease. Circ J 104(7): 796-801.

Wathen MS, DeGroot PJ, Sweeney MO, Stark AJ, Otterness MF, Adkisson WO, Canby RC, Khalighi K, Machado C, Rubenstein DS, et al. (2004): Prospective randomized multi- 
center trial of empirical antitachycardia pacing versus shocks for spontaneous rapid ventricular tachycardia in patients with implantable cardioverter-defibrillators: Pacing Fast Ventricular Tachycardia Reduces Shock Therapies (PainFREE Rx II) trial results. Circulation 110(17): 2591-2596.

Willems S, Eckardt L, Hoffmann E, Klemm H, Pitschner HF, Reithmann C, Tebbenjohanns J, Zrenner B (2007): Leitlinie invasive elektrophysiologische Diagnostik. Clin Res Cardiol $\underline{96}(9): 634-651$.

Wilson D, Shi B, Harding S, Lever N, Larsen P (2012): Implantable cardioverterdefibrillators: a long-term view. Intern Med J 42 (5): 554-561.

Wollmann G, Böcker D, Löher A, Scheld HH, Breithardt G, Gradaus R (2008): Diagnostik und Therapie von ICD-Elektrodendefekten. Herzschr Elektrophys 19(4): 169-180.

Zipes DP, Camm AJ, Borggrefe M, Buxton AE, Chaitman B, Fromer M, Gregoratos G, Klein G, Moss AJ, Myerburg RJ, et al. (2006): ACC/AHA/ESC 2006 Guidelines for Management of Patients With Ventricular Arrhythmias and the Prevention of Sudden Cardiac Death: a report of the American College of Cardiology/American Heart Association Task Force and the European Society of Cardiology Committee for Practice Guidelines (writing committee to develop Guidelines for Management of Patients With Ventricular Arrhythmias and the Prevention of Sudden Cardiac Death): developed in collaboration with the European Heart Rhythm Association and the Heart Rhythm Society. Circulation 114(10): e385-484. 


\section{Danksagung}

Zunächst möchte ich mich bei Herrn Professor Dr. med. Markus Zabel für die freundliche Überlassung des Themas und für das große persönliche Engagement bei der Betreuung meiner Arbeit bedanken.

Besonderen Dank schulde ich Herrn Dr. med. Joachim Seegers, der mir durch seine engagierte Betreuung und stete Diskussionsbereitschaft vielseitige Denkanstöße gegeben und mich bei der Erstellung dieser Arbeit maßgeblich unterstützt hat.

Des Weiteren danke ich allen Mitarbeiterinnen und Mitarbeitern des Herzzentrums Göttingen, insbesondere der Abteilung Elektrophysiologie, für die ausgesprochen angenehme Zusammenarbeit und Hilfsbereitschaft.

Den Mitarbeitern der Abteilung Medizinische Statistik danke ich für ihre Hilfe bei der statistischen Analyse der Ergebnisse. 